\author{
CHAPTER 5
}

\title{
Draw Solutes in Forward Osmosis Processes
}

\author{
Ho Kyong Shon, Laura Chekli, Sherub Phuntsho, Jungeun Kim and \\ Jaeweon Cho
}

This chapter aims to provide valuable insight for the selection of suitable draw solution (DS) and review different DS characteristics affecting the performance of forward osmosis (FO) processes. It also presents a comprehensive classification of the different DS that have been tested so far. Although there are some commercial applications of FO technology, the development of an effective large-scale process is currently limited due to the lack of both suitable DS and membrane. The main challenge regarding the development of appropriate DS is their separation and recovery from the product water, especially for drinking water applications. The recovery process should not be expensive and energy intensive otherwise the FO process cannot be advantageous compared with other membrane technologies such as RO.

\subsection{General Criteria for Selection of Suitable DS}

The selection of a suitable DS is crucial for the development of the FO technology and will not only improve the performance of the process but also save significant costs regarding the separation and recovery process. The selection of appropriate DS include an initial screening based on the intrinsic properties of the DS, laboratory-scale performance, full-scale performance and finally commercialisation as depicted in Figure 5.1. The information provided remains general and applicable to any applications. However, it is obvious that further specific criteria, depending on the targeted application, also need to be evaluated.

Initial screening can be carried out using thermodynamic modelling softwares such as OLI Stream Analyzer. These softwares can be very useful to calculate some basic intrinsic properties including water solubility, $\mathrm{pH}$, speciation and osmotic pressure, which are important properties that affect the performance of the FO process (see section 5.2 for more details). It is also essential to ensure that the DS is inert, of near neutral $\mathrm{pH}$, 
stable and have minimal toxicity, especially if the targeted application is the production of drinking water. In that specific case, the DS must be safe for human consumption. International norms on water quality and human health can be found on the WHO guidelines for drinking-water quality (WHO 2011). Another important criterion to evaluate is the compatibility between the DS and the FO membrane. In fact, the selected DS should not modify the properties and performance of the membrane through fouling, reaction, adsorption or dissolution. Finally, the DS should be produced at low cost to ensure the economic viability of the process.

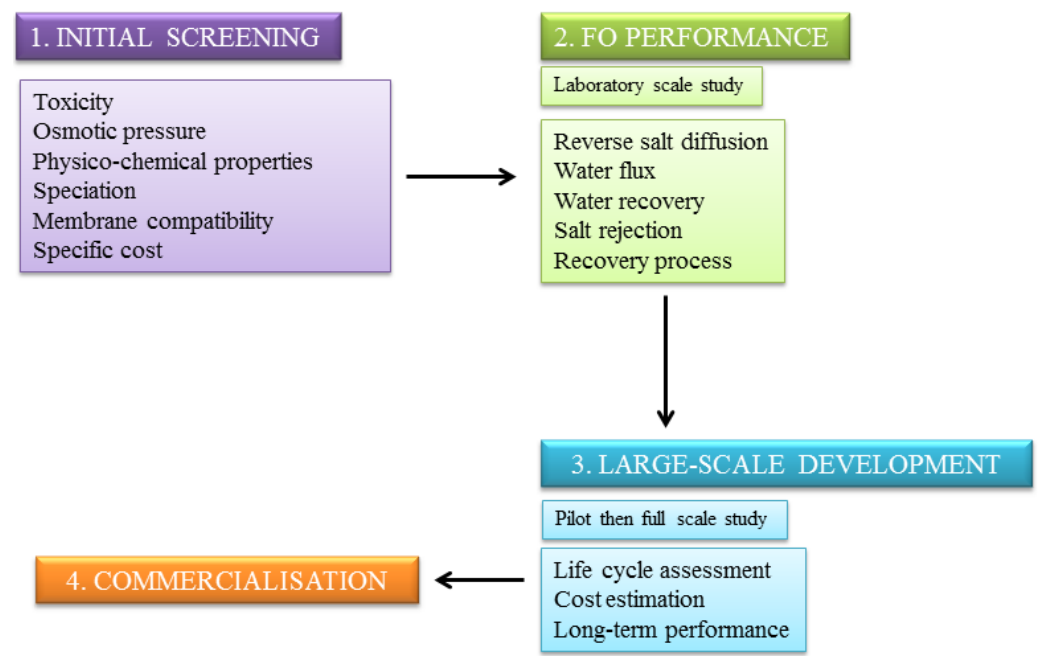

Figure 5.1 General criteria for selection of suitable DS

Once the preliminary selection has been done, laboratory experiments can be conducted to evaluate the performance of the pre-selected DS in terms of reverse salt diffusion (RSF), water flux and water recovery, which are the main parameters used to assess the performance of the FO process. In the case of desalination applications, salt rejection is also an essential parameter to consider. Another crucial parameter to evaluate in most applications is the recovery process of the DS. This process should not be complex and be able to reconcentrate and recover the DS at low-energy cost.

Finally, the remaining DS should be tested at full-scale, and life cycle assessment should be conducted to assess the environmental impacts of each stage of the process (i.e. from the production of raw materials to the treatment and disposal of waste).

\subsection{DS Characteristics Affecting FO Performance}

There are several factors affecting the FO process performance, and these factors are generally associated to the membrane properties, DS characteristics and operating 
conditions. Some recent studies investigated the effect of these different parameters on the process performance (Xu et al. 2010; Zhao and Zou 2011; Zhao et al. 2011; Lay et al. 2012; Mamisaheby et al. 2012). This section will focus on the DS characteristics.

\subsubsection{General DS Characteristics}

As the osmotic pressure across the FO membrane is the driving force of the process, the DS must exhibit high solubility in water and high osmotic pressure, much higher than the osmotic pressure of the feed solution in order to induce high water flux

across the membrane. Based on the theory proposed by Van't Hoff (1887), the osmotic pressure $(\pi)$ of an ideal dilute solution is expressed as follow:

$$
\pi=n\left(\frac{c}{M}\right) R T
$$

where $\mathrm{n}$ is the number of moles of species formed by the dissociation of solutes in the solution; c is the concentration of the solute in $\mathrm{g} / \mathrm{L} ; \mathrm{M}$ is the molecular weight of the solute in $\mathrm{g} / \mathrm{mol}$; $\mathrm{R}$ is the gas constant $(\mathrm{R}=0.0821)$; and $\mathrm{T}$ is the absolute temperature of the solution in K. Based on equation (5.1), it is clear that a DS combining a low molecular weight and high water solubility will produce a high osmotic pressure (on equal mass basis) and generate high water flux across the membrane (McCutcheon et al. 2005).

The diffusion coefficient $\left(D_{s}\right)$ of the DS will also affect the performance of the process and can be described as (Van't Hoff 1887):

$$
K=\frac{t \tau}{\varepsilon D_{S}}
$$

where $K$ is the solute resistance to diffusion within the membrane support layer, $t, \tau$ and $\varepsilon$ represent the thickness, tortuosity and porosity of the membrane support layer respectively. This equation clearly shows that a DS with a high diffusion coefficient will readily diffuse through the membrane support layer and thus decrease the ICP effects. In general, solutes with lower molecular weight exhibit higher diffusion coefficient and can thus theoretically promote higher water fluxes across the membrane. However, low molecular weight solutes also show high RSF (Ng and Tang 2006; Achilli et al. 2010; Wang et al. 2010; Yen et al. 2010; Phuntsho et al. 2011) and several studies demonstrated that this phenomenon can jeopardise the process (Hancock and Cath 2009; Lee et al. 2010). The reverse salt diffusion $\left(J_{S}\right)$ of a DS can be calculated from the increase of the DS concentration in the feed solution:

$$
J_{s}=\frac{\left(C_{t} V_{t}\right)-\left(C_{0} V_{0}\right)}{\Delta t} \frac{1}{A_{m}}
$$


where $C_{t}(\mathrm{~mol} / \mathrm{L})$ and $V_{t}(\mathrm{~L})$ are the salt concentration and feed volume at a time t, $\mathrm{C}_{0}$ $(\mathrm{mol} / \mathrm{L})$ and $\mathrm{V}_{0}(\mathrm{~L})$ are the salt concentration and feed volume at $\mathrm{t}_{0}, \Delta t=t-t_{0}(\mathrm{~h})$ is the duration of the experiment and $A_{m}\left(\mathrm{~m}^{2}\right)$ is the effective membrane area. When DS exhibit electrical conductivities in aqueous solution, their concentration in the feed solution can be easily monitored when pure water is used as feed solution. RSF of the DS can not only decrease the performance of the process by decreasing the net osmotic pressure across the membrane, but can also complicate concentrate management. The accumulation of DS in the feed solution can also have adverse effect on sensitive receiving environment or alter adjacent treatment processes (Hancock and Cath 2009).

As for any pressure-based membrane process, temperature is also affecting the performance of the FO process; and more specifically, the DS temperature may impact significantly on the efficiency of the process. Many studies have demonstrated that increasing the DS temperature enhanced the water flux due to reduced water viscosity and thus improved mass transfer across the membrane (Petrotos et al. 1998; McCutcheon et al. 2006; Garcia-Castello et al. 2009; Zhao and Zou 2011). Increasing the DS solution temperature will also increase its diffusion coefficient in solution and therefore decrease the value of $\mathrm{K}$ (i.e. solute resistance to diffusion within the membrane support layer) based on equation (2) which will ultimately improve the water flux (Petrotos et al. 1998; McCutcheon et al. 2005). However, it was also demonstrated recently that increasing temperature improved water flux only to a certain critical point; after which membrane scaling occurred, causing flux decline and in overall decreasing the process performance (Garcia-Castello et al. 2009; Zhao and Zou 2011).

Finally, the DS concentration has also been shown to influence the process performance (McCutcheon et al. 2006; Achilli et al. 2009; Choi et al. 2009; Xu et al. 2010). However, the relationship between DS concentration and water flux is not linear, especially at high DS concentration where the relation has been observed to become logarithmic. This has been attributed to ICP effects in the membrane support layer which become more important at higher permeate flux resulting in less effective water flux improvement (Tan and Ng 2010).

\subsubsection{Other DS Characteristics}

Other specific DS characteristics can impact on the process performance depending on the targeted application. For instance, Achilli et al. (2010) demonstrated that the use of DS containing scale precursor ions (e.g. $\mathrm{Mg}^{2+}, \mathrm{Ca}^{2+}, \mathrm{Ba}^{2+}, \mathrm{SO}_{4}{ }^{2-}$ and $\mathrm{CO}_{3}{ }^{2-}$ ) has to be limited to application involving the use of pure water as feed solution such as the food industry. In fact, the presence of scale precursor ions will promote mineral scaling on the membrane surface when the feed solution concentration is above the solubility limit. Novel synthetic DS have been recently developed and their unique properties may also impact on the process efficiency. For instance, magnetic 
nanoparticles (MNPs) as DS, have been developed by Chung and co-workers (Ling et al. 2010; Ling and Chung 2011b; Ling and Chung 2011a; Ling et al. 2011) and demonstrated a strong tendency to aggregate which could enhance membrane fouling and decrease the performance of the process.

\subsection{Classification of Osmotic DS}

In the past few decades, there has been a rapid progress in the development of suitable draw solutions for FO process; however, only few reviews on draw solutions have been published to date (Chekli et al. 2012; Ge et al. 2013). Draw solutions can be generally classified as volatile compounds, organic compounds, inorganic compounds and novel synthetic compounds including MNPs and polymer hydrogel. Subclassification may include electrolyte and non-electrolyte solutions depending on the surface charge of the DS. An overview of the different DS that has been tested so far, including some of their properties and performance is summarised in Table 5.1.

\subsubsection{Volatile Compounds}

The development of volatile compounds as draw solute started in the early 60's. Neff (1964) was the first to propose a mixture of ammonia and carbon dioxide gases for seawater desalination. In the patent, the experimental conditions to prepare ammonium bicarbonate $\left(\mathrm{NH}_{4} \mathrm{HCO}_{3}\right)$ solution and its regeneration process (i.e. a gas-liquid separating column) are explained. However, due to its low solubility in water, $\mathrm{NH}_{4} \mathrm{HCO}_{3}$ produced a low osmotic pressure, limited its application for FO process. To overcome this issue, McGinnis and co-workers (McCutcheon et al. 2006; McGinnis et al. 2007) developed a novel thermolyte solution using a mixture of ammonium hydroxide $\left(\mathrm{NH}_{4} \mathrm{OH}\right)$ and $\mathrm{NH}_{4} \mathrm{HCO}_{3}$ in specific proportion or by fluctuating the ratio of ammonia and carbon dioxide for the preparation of $\mathrm{NH}_{4} \mathrm{HCO}_{3}$ to increase its solubility. Higher osmotic pressure was achieved (i.e. up to 238 bars) as well as high water fluxes, close to $25 \mathrm{LMH}$. They also demonstrated that the mixture can be easily recovered by distillation process using low heat energy as this DS can decompose into $\mathrm{NH}_{3}$ and $\mathrm{CO}_{2}$ by heating up to only $60{ }^{\circ} \mathrm{C}$ (McCutcheon et al. 2006). However, the proximity of low-grade heat (e.g. from thermal power plants) is required to ensure that the recovery process is economically viable. The use of $\mathrm{NH}_{4} \mathrm{HCO}_{3}$ as DS was also investigated in other recent studies for desalination application (Ng and Tang 2006; Hancock and Cath 2009; Achilli et al. 2010). The solubility of $\mathrm{NH}_{4} \mathrm{HCO}_{3}$ was found to be the lowest in comparison to other tested DS and its RSF was among the highest due its low molecular weight (Achilli et al. 2010). Ng and Tang (2006) also found that this DS can suffer from early decomposition if the operated temperature is higher than $30{ }^{\circ} \mathrm{C}$. 
Table 5.1 Overview of the different draw solutions used in FO process.

\begin{tabular}{|c|c|c|c|c|c|c|c|c|}
\hline $\begin{array}{c}\text { Draw } \\
\text { solution } \\
\text { types } \\
\end{array}$ & Draw solution & $\begin{array}{c}\text { Osmotic } \\
\text { pressure } \\
\text { (concentration) } \\
\end{array}$ & $\begin{array}{c}\text { Water flux } \\
\text { (Feed solution } \\
\text { - membrane) } \\
\end{array}$ & $\begin{array}{l}\text { Reverse salt } \\
\quad \text { flux }\end{array}$ & $\begin{array}{l}\text { Recovery } \\
\text { process(es) }\end{array}$ & Drawbacks & $\begin{array}{l}\text { Example(s) of } \\
\text { application }\end{array}$ & $\begin{array}{l}\text { Commercial } \\
\text { status }\end{array}$ \\
\hline \multirow[t]{2}{*}{$\begin{array}{l}\text { Volatile } \\
\text { compounds }\end{array}$} & $\begin{array}{l}\text { Ammonium } \\
\text { Bicarbonate } \\
\left(\mathrm{NH}_{4} \mathrm{HCO}_{3}\right)\end{array}$ & $\begin{array}{c}66.40 \mathrm{~atm} \\
(2.0 \mathrm{M})\end{array}$ & $\begin{array}{c}2.04 \mu \mathrm{m} / \mathrm{s} \\
\text { (DI water - CTA } \\
\text { flat sheet) }\end{array}$ & $\begin{array}{l}18.2 \mathrm{~g} / \mathrm{m}^{2} . h \\
\text { at } 2.8 \mathrm{MPa}\end{array}$ & $\begin{array}{c}\text { Heating - } \\
\text { decomposition } \\
\text { into } \mathrm{NH}_{3} \text { and } \mathrm{CO}_{2}\end{array}$ & $\begin{array}{l}\text { Low solubility in } \\
\text { water; High reverse } \\
\text { salt diffusion; High } \\
\text { replenishment cost; } \\
\text { Not thermally stable }\end{array}$ & $\begin{array}{l}\text { Seawater desalination } \\
\text { (McCutcheon et al. } \\
\text { 2005; McCutcheon et al. } \\
\text { 2006; Ng et al. 2006; } \\
\text { Hancock and Cath 2009; } \\
\text { Achilli et al. 2010) }\end{array}$ & $\begin{array}{l}\text { Commercial } \\
\text { (Oasys Water) } \\
\text { for brine } \\
\text { concentration }\end{array}$ \\
\hline & $\begin{array}{l}\text { Sulfur dioxide } \\
\qquad\left(\mathrm{SO}_{2}\right)\end{array}$ & Not reported & Not reported & Not reported & $\begin{array}{l}\text { Heating, air } \\
\text { stripping or } \\
\text { distillation }\end{array}$ & $\begin{array}{l}\text { Volatile; Corrosive; } \\
\text { Unstable in solution }\end{array}$ & $\begin{array}{c}\text { Seawater desalination } \\
\text { (Batchelder 1965; Glew } \\
\text { 1965) }\end{array}$ & - \\
\hline \multirow{3}{*}{$\begin{array}{l}\text { Organic } \\
\text { compounds }\end{array}$} & $\begin{array}{l}\text { Glucose } \\
\left(\mathrm{C}_{6} \mathrm{H}_{12} \mathrm{O}_{6}\right)\end{array}$ & $\begin{array}{l}55.03 \mathrm{~atm} \\
(2.0 \mathrm{M})\end{array}$ & $\begin{array}{l}0.37 \mathrm{LMH} \\
\text { (tomato juice - } \\
\text { thin film } \\
\text { composite } \\
\text { aromatic } \\
\text { polyamide) }\end{array}$ & Not reported & $\begin{array}{l}\text { Direct application, } \\
\text { Low pressure RO }\end{array}$ & \multirow{2}{*}{$\begin{array}{l}\text { High molecular size } \\
\text { causes high internal } \\
\text { concentration } \\
\text { polarization (ICP) } \\
\text { effect }\end{array}$} & $\begin{array}{c}\text { Emergency drinking } \\
\text { solutions (Kravath and } \\
\text { Davis 1975; Wallace et } \\
\text { al. 2008); Seawater } \\
\text { desalination (Yaeli 1992; } \\
\text { Ng and Tang 2006); } \\
\text { Tomato juice } \\
\text { concentration (Petrotos } \\
\text { et al. 1998) }\end{array}$ & - \\
\hline & $\begin{array}{l}\text { Fructose } \\
\left(\mathrm{C}_{6} \mathrm{H}_{12} \mathrm{O}_{6}\right)\end{array}$ & $\begin{array}{c}55.02 \mathrm{~atm} \\
(2.0 \mathrm{M})\end{array}$ & $\begin{array}{c}2.5 \mathrm{LMH} \\
(0.5 \mathrm{M} \mathrm{NaCl}- \\
\text { thin layer of } \\
\text { cotton-derived } \\
\text { cellulose-ester } \\
\text { plastics } \\
\text { embedded on } \\
\text { top of a } \\
\text { microfiltration } \\
\text { membrane) } \\
\end{array}$ & Not reported & Direct application & & $\begin{array}{l}\text { Nutritious drink } \\
\text { production (Stache } \\
\text { 1989); Seawater } \\
\text { desalination (Ng et al. } \\
\text { 2006); Brine control (Ng } \\
\text { and Tang 2006) }\end{array}$ & - \\
\hline & $\begin{array}{l}\text { Sucrose } \\
\left(\mathrm{C}_{12} \mathrm{H}_{22} \mathrm{O}_{11}\right)\end{array}$ & $\begin{array}{c}26.7 \mathrm{~atm} \\
(1.0 \mathrm{M})\end{array}$ & $\begin{array}{c}12.9 \mathrm{LMH} \\
\text { (DI water - CA } \\
\text { hollow fibre) }\end{array}$ & Not reported & Nanofiltration & Low water flux & $\begin{array}{l}\text { Wastewater treatment } \\
\quad \text { (Su et al. 2012) }\end{array}$ & - \\
\hline
\end{tabular}




\begin{tabular}{|c|c|c|c|c|c|c|c|c|}
\hline $\begin{array}{l}\text { Draw } \\
\text { solution } \\
\text { types }\end{array}$ & Draw solution & $\begin{array}{c}\text { Osmotic } \\
\text { pressure } \\
\text { (concentration) }\end{array}$ & $\begin{array}{c}\text { Water flux } \\
\text { (Feed solution } \\
\text { - membrane) }\end{array}$ & $\begin{array}{l}\text { Reverse salt } \\
\text { flux }\end{array}$ & $\begin{array}{l}\text { Recovery } \\
\text { process(es) }\end{array}$ & Drawbacks & $\begin{array}{l}\text { Example(s) of } \\
\text { application }\end{array}$ & $\begin{array}{l}\text { Commercial } \\
\text { status }\end{array}$ \\
\hline \multirow{6}{*}{$\begin{array}{l}\text { Organic } \\
\text { compounds }\end{array}$} & $\begin{array}{l}\text { Ethanol } \\
\left(\mathrm{C}_{2} \mathrm{H}_{6} \mathrm{O}\right)\end{array}$ & $\begin{array}{l}49.93 \mathrm{~atm} \\
(2.0 \mathrm{M})\end{array}$ & Not reported & Not reported & $\begin{array}{l}\text { Pervaporation- } \\
\text { based separation }\end{array}$ & $\begin{array}{l}\text { High reverse salt } \\
\text { diffusion and low } \\
\text { water flux }\end{array}$ & $\begin{array}{l}\text { Recovery of water from } \\
\text { highly impaired sources } \\
\text { (McCormick et al. 2008) }\end{array}$ & - \\
\hline & $\begin{array}{l}\text { Sodium formate } \\
\text { (HCOONa) }\end{array}$ & $\begin{array}{c}31.4 \mathrm{MPa} \\
\text { (at saturation) }\end{array}$ & $\begin{array}{c}2.6 \mu \mathrm{m} / \mathrm{s} \text { at } 2.8 \\
\mathrm{MPa} \\
\text { (DI water }-\mathrm{CTA} \\
\text { flat sheet) }\end{array}$ & $\begin{array}{l}6.04 \mathrm{~g} / \mathrm{m}^{2} . \mathrm{h} \\
\text { at } 2.8 \mathrm{MPa}\end{array}$ & \multirow{4}{*}{ RO process } & $\begin{array}{l}\text { High reverse salt flux; } \\
\text { High replenishment } \\
\text { cost }\end{array}$ & \multirow{4}{*}{$\begin{array}{l}\text { Wastewater treatment } \\
\text { (Bowden et al. 2012) }\end{array}$} & - \\
\hline & $\begin{array}{l}\text { Sodium acetate } \\
\qquad\left(\mathrm{C}_{2} \mathrm{H}_{3} \mathrm{NaO}_{2}\right)\end{array}$ & $\begin{array}{c}27.0 \mathrm{MPa} \\
\text { (at saturation) }\end{array}$ & $\begin{array}{c}2.5 \mu \mathrm{m} / \mathrm{s} \text { at } 2.8 \\
\mathrm{MPa} \\
\text { (DI water - CTA } \\
\text { flat sheet) }\end{array}$ & $\begin{array}{l}2.73 \mathrm{~g} / \mathrm{m}^{2} . \mathrm{h} \\
\text { at } 2.8 \mathrm{MPa}\end{array}$ & & \multirow{3}{*}{$\begin{array}{l}\text { Relatively high } \\
\text { replenishment cost } \\
\text { compared to inorganic } \\
\text { salts }\end{array}$} & & \multirow{3}{*}{ - } \\
\hline & $\begin{array}{l}\text { Sodium } \\
\text { propionate } \\
\left(\mathrm{C}_{3} \mathrm{H}_{5} \mathrm{NaO}_{2}\right)\end{array}$ & N.A. & $\begin{array}{c}2.8 \mathrm{MPa} \\
\text { (DI water - CTA } \\
\text { flat sheet) }\end{array}$ & $\begin{array}{l}1.47 \mathrm{~g} / \mathrm{m}^{2} . \mathrm{h} \\
\text { at } 2.8 \mathrm{MPa}\end{array}$ & & & & \\
\hline & $\begin{array}{c}\text { Magnesium } \\
\text { acetate } \\
\left(\mathrm{Mg}\left(\mathrm{CH}_{3} \mathrm{COO}\right)_{2}\right)\end{array}$ & $\begin{array}{c}10.3 \mathrm{MPa} \\
\text { (at saturation) }\end{array}$ & $\begin{array}{c}2.25 \mu \mathrm{m} / \mathrm{s} \text { at } \\
2.8 \mathrm{MPa} \\
\text { (DI water - CTA } \\
\text { flat sheet) }\end{array}$ & $\begin{array}{l}1.07 \mathrm{~g} / \mathrm{m}^{2} . \mathrm{h} \\
\text { at } 2.8 \mathrm{MPa}\end{array}$ & & & & \\
\hline & $\begin{array}{l}\text { Switchable } \\
\text { polarity solvents } \\
\text { (SPS) }\end{array}$ & $\begin{array}{l}\sim 325 \mathrm{~atm} \\
(7.6 \mathrm{~mol} / \mathrm{kg})\end{array}$ & $\begin{array}{c}10 \mathrm{LMH} \text { at } 7.6 \\
\mathrm{~mol} / \mathrm{kg} \mathrm{(2} \\
\mathrm{mol} / \mathrm{kg} \mathrm{NaCl}- \\
\text { CTA flat sheet) }\end{array}$ & $\begin{array}{l}1.8 \text { wt. \% of } \\
\text { the initial DS } \\
\text { concentration }\end{array}$ & $\begin{array}{l}\text { Polar to non-polar } \\
\text { phase shift } \\
\text { induced by } \mathrm{CO}_{2}\end{array}$ & $\begin{array}{l}\text { Degradation of FO } \\
\text { membrane }\end{array}$ & $\begin{array}{l}\text { Industrial purification of } \\
\text { water (Stone et al. } \\
\text { 2013a) }\end{array}$ & $\begin{array}{c}\text { Under } \\
\text { development } \\
\text { (Idaho } \\
\text { National } \\
\text { Laboratory) }\end{array}$ \\
\hline
\end{tabular}




\begin{tabular}{|c|c|c|c|c|c|c|c|c|}
\hline $\begin{array}{l}\text { Draw } \\
\text { solution } \\
\text { types }\end{array}$ & Draw solution & $\begin{array}{c}\text { Osmotic } \\
\text { pressure } \\
\text { (concentration) }\end{array}$ & $\begin{array}{c}\text { Water flux } \\
\text { (Feed solution } \\
\text { - membrane) }\end{array}$ & $\begin{array}{l}\text { Reverse salt } \\
\text { flux }\end{array}$ & $\begin{array}{l}\text { Recovery } \\
\text { process(es) }\end{array}$ & Drawbacks & $\begin{array}{l}\text { Example(s) of } \\
\text { application }\end{array}$ & $\begin{array}{l}\text { Commercial } \\
\text { status }\end{array}$ \\
\hline \multirow{6}{*}{$\begin{array}{l}\text { Inorganic } \\
\text { salts }\end{array}$} & $\begin{array}{l}\text { Sodium Chloride } \\
\text { ( } \mathrm{NaCl})\end{array}$ & $\begin{array}{c}100.40 \mathrm{~atm} \\
(2.0 \mathrm{M})\end{array}$ & $\begin{array}{c}2.68 \mu \mathrm{m} / \mathrm{s} \\
\text { (DI water - CTA } \\
\text { flat sheet) }\end{array}$ & $\begin{array}{c}7.2 \mathrm{~g} / \mathrm{m}^{2} . \mathrm{h} \text { at } \\
2.8 \mathrm{MPa}\end{array}$ & $\begin{array}{c}\text { RO process, } \\
\text { Distillation/RO, } \\
\text { Direct application }\end{array}$ & $\begin{array}{l}\text { High reverse salt } \\
\text { diffusion; Protein } \\
\text { deterioration }\end{array}$ & $\begin{array}{c}\text { Algal biodiesel } \\
\text { production (Zou et al. } \\
\text { 2011); Seawater } \\
\text { desalination (Achilli et } \\
\text { al. 2010); Sucrose } \\
\text { concentration (Garcia- } \\
\text { Castello et al. 2009); } \\
\text { Reuse of domestic } \\
\text { wastewater (Achilli et al. } \\
\text { 2009); Juice } \\
\text { concentration (Petrotos } \\
\text { et al. 1998); Emergency } \\
\text { drinking solutions } \\
\text { (Wallace et al. 2008) }\end{array}$ & $\begin{array}{c}\text { Pre- } \\
\text { commercial } \\
\text { (IDE } \\
\text { Technologies, } \\
\text { Porifera) }\end{array}$ \\
\hline & $\begin{array}{l}\text { Potassium } \\
\text { Chloride } \\
\text { (KCl) }\end{array}$ & $\begin{array}{c}89.30 \mathrm{~atm} \\
(2.0 \mathrm{M})\end{array}$ & $\begin{array}{c}6.337 \mu \mathrm{m} / \mathrm{s} \\
\text { (DI water - CA } \\
\text { embedded in } \\
\text { polyester } \\
\text { woven mesh) }\end{array}$ & $\begin{array}{c}0.222 \\
\mathrm{mmoles} / \mathrm{m}^{2} . \mathrm{s}\end{array}$ & Direct application & $\begin{array}{l}\text { High reverse salt } \\
\text { diffusion }\end{array}$ & $\begin{array}{c}\text { Direct fertigation } \\
\text { (Phuntsho et al. 2011); } \\
\text { Desalination (Achilli et } \\
\text { al. 2010) }\end{array}$ & - \\
\hline & $\begin{array}{l}\text { Ammonium } \\
\text { Chloride } \\
\left(\mathrm{NH}_{4} \mathrm{Cl}\right)\end{array}$ & $\begin{array}{c}87.70 \mathrm{~atm} \\
(2.0 \mathrm{M})\end{array}$ & $\begin{array}{c}5.348 \mu \mathrm{m} / \mathrm{s} \\
\text { (DI water - CA } \\
\text { embedded in } \\
\text { polyester } \\
\text { woven mesh) }\end{array}$ & $\begin{array}{c}0.333 \\
\mathrm{mmoles} / \mathrm{m}^{2} . \mathrm{s}\end{array}$ & Direct application & $\begin{array}{l}\text { High reverse salt } \\
\text { diffusion }\end{array}$ & $\begin{array}{c}\text { Direct fertigation } \\
\text { (Phuntsho et al. 2011); } \\
\text { Desalination (Achilli et } \\
\text { al. 2010) }\end{array}$ & - \\
\hline & $\begin{array}{l}\text { Ammonium } \\
\text { Nitrate } \\
\left(\mathrm{NH}_{4} \mathrm{NO}_{3}\right)\end{array}$ & $\begin{array}{l}64.90 \mathrm{~atm} \\
(2.0 \mathrm{M})\end{array}$ & $\begin{array}{c}4.177 \mu \mathrm{m} / \mathrm{s} \\
\text { (DI water - CA } \\
\text { embedded in } \\
\text { polyester } \\
\text { woven mesh) }\end{array}$ & $\begin{array}{c}0.791 \\
\mathrm{mmoles} / \mathrm{m}^{2} . \mathrm{s}\end{array}$ & Direct application & $\begin{array}{l}\text { High reverse salt } \\
\text { diffusion }\end{array}$ & $\begin{array}{l}\text { Direct fertigation } \\
\text { (Phuntsho et al. 2011) }\end{array}$ & - \\
\hline & $\begin{array}{l}\text { Potassium } \\
\text { Bromide } \\
\text { (KBr) }\end{array}$ & $\begin{array}{l}89.70 \mathrm{~atm} \\
(2.0 \mathrm{M})\end{array}$ & $\begin{array}{c}2.84 \mu \mathrm{m} / \mathrm{s} \\
\text { (DI water - CTA } \\
\text { flat sheet) }\end{array}$ & $\begin{array}{c}22 \mathrm{~g} / \mathrm{m}^{2} . \mathrm{h} \text { at } \\
2.8 \mathrm{MPa}\end{array}$ & RO process & $\begin{array}{l}\text { Very high reverse salt } \\
\text { diffusion; High } \\
\text { replenishment cost }\end{array}$ & $\begin{array}{c}\text { Desalination } \\
\text { (Achilli et al. 2010) }\end{array}$ & - \\
\hline & $\begin{array}{c}\text { Sodium } \\
\text { Bicarbonate } \\
\left(\mathrm{NaHCO}_{3}\right)\end{array}$ & $\begin{array}{l}46.70 \mathrm{~atm} \\
(2.0 \mathrm{M})\end{array}$ & $\begin{array}{c}2.47 \mu \mathrm{m} / \mathrm{s} \\
\text { (DI water - CTA } \\
\text { flat sheet) }\end{array}$ & $\begin{array}{c}1.7 \mathrm{~g} / \mathrm{m}^{2} . \mathrm{h} \text { at } \\
2.8 \mathrm{MPa}\end{array}$ & RO process & $\begin{array}{l}\text { Low water solubility; } \\
\text { Contain scale } \\
\text { precursor ions; }\end{array}$ & $\begin{array}{c}\text { Desalination } \\
\text { (Achilli et al. 2010) }\end{array}$ & - \\
\hline
\end{tabular}




\begin{tabular}{|c|c|c|c|c|c|c|c|c|}
\hline $\begin{array}{c}\text { Draw } \\
\text { solution } \\
\text { types }\end{array}$ & Draw solution & $\begin{array}{c}\text { Osmotic } \\
\text { pressure } \\
\text { (concentration) } \\
\end{array}$ & $\begin{array}{c}\text { Water flux } \\
\text { (Feed solution } \\
\text { - membrane) } \\
\end{array}$ & $\begin{array}{l}\text { Reverse salt } \\
\text { flux }\end{array}$ & $\begin{array}{l}\text { Recovery } \\
\text { process(es) }\end{array}$ & Drawbacks & $\begin{array}{l}\text { Example(s) of } \\
\text { application }\end{array}$ & $\begin{array}{l}\text { Commercial } \\
\text { status }\end{array}$ \\
\hline \multirow{8}{*}{$\begin{array}{l}\text { Inorganic } \\
\text { salts }\end{array}$} & $\begin{array}{l}\text { Potassium } \\
\text { Bicarbonate } \\
\left(\mathrm{KHCO}_{3}\right)\end{array}$ & $\begin{array}{l}79.30 \mathrm{~atm} \\
(2.0 \mathrm{M})\end{array}$ & $\begin{array}{c}2.25 \mu \mathrm{m} / \mathrm{s} \\
\text { (DI water - CTA } \\
\text { flat sheet) }\end{array}$ & $\begin{array}{c}1.4 \mathrm{~g} / \mathrm{m}^{2} . \mathrm{h} \text { at } \\
2.8 \mathrm{MPa}\end{array}$ & RO process & $\begin{array}{c}\text { Contain scale } \\
\text { precursor ions; Not } \\
\text { easily recovered by RO }\end{array}$ & $\begin{array}{c}\text { Desalination } \\
\text { (Achilli et al. 2010) }\end{array}$ & - \\
\hline & $\begin{array}{l}\text { Magnesium } \\
\text { Chloride } \\
\left(\mathrm{MgCl}_{2}\right)\end{array}$ & $\begin{array}{l}256.50 \mathrm{~atm} \\
(2.0 \mathrm{M})\end{array}$ & $\begin{array}{c}2.33 \mu \mathrm{m} / \mathrm{s} \\
\text { (DI water - CTA } \\
\text { flat sheet) }\end{array}$ & $\begin{array}{c}4.8 \mathrm{~g} / \mathrm{m}^{2} . h \text { at } \\
2.8 \mathrm{MPa}\end{array}$ & $\begin{array}{l}\text { Nanofiltration or } \\
\text { direct application }\end{array}$ & $\begin{array}{l}\text { High viscosity; Low } \\
\text { diffusion coefficient; } \\
\mathrm{Mg}^{2+} \text { may affect } \\
\text { membrane fouling by } \\
\text { complexing with some } \\
\text { functional group }\end{array}$ & $\begin{array}{c}\text { Seawater desalination } \\
\text { (Hancock and Cath 2009; } \\
\text { Achilli et al. 2010); } \\
\text { Emergency drinking } \\
\text { solutions (Wallace et al. } \\
\text { 2008); Algal biodiesel } \\
\text { production (Zou et al. } \\
\text { 2011) } \\
\end{array}$ & - \\
\hline & $\begin{array}{l}\text { Calcium Chloride } \\
\qquad\left(\mathrm{CaCl}_{2}\right)\end{array}$ & $\begin{array}{l}217.60 \mathrm{~atm} \\
(2.0 \mathrm{M})\end{array}$ & $\begin{array}{c}2.64 \mu \mathrm{m} / \mathrm{s} \\
\text { (DI water - CTA } \\
\text { flat sheet) }\end{array}$ & $\begin{array}{c}7.9 \mathrm{~g} / \mathrm{m}^{2} . h \text { at } \\
2.8 \mathrm{MPa}\end{array}$ & RO process & $\begin{array}{l}\text { Contain scale } \\
\text { precursor ions }\end{array}$ & $\begin{array}{c}\text { Tomato juice } \\
\text { concentration (Petrotos } \\
\text { et al. 1998); Emergency } \\
\text { drinking solutions } \\
\text { (Wallace et al. 2008); } \\
\text { Seawater desalination } \\
\text { (Achilli et al. 2010) } \\
\end{array}$ & - \\
\hline & $\begin{array}{l}\text { Ammonium } \\
\text { Sulfate } \\
\left(\left(\mathrm{NH}_{4}\right)_{2} \mathrm{SO}_{4}\right)\end{array}$ & $\begin{array}{l}92.10 \mathrm{~atm} \\
(2.0 \mathrm{M})\end{array}$ & $\begin{array}{c}5.391 \mu \mathrm{m} / \mathrm{s} \\
\text { (DI water - CA } \\
\text { embedded in } \\
\text { polyester } \\
\text { woven mesh) } \\
\end{array}$ & $\begin{array}{c}0.006 \\
\mathrm{mmoles} / \mathrm{m}^{2} . \mathrm{s}\end{array}$ & Direct application & $\begin{array}{l}\text { High replenishment } \\
\text { cost }\end{array}$ & $\begin{array}{c}\text { Direct fertigation } \\
\text { (Phuntsho et al. 2011); } \\
\text { Desalination (Achilli et } \\
\text { al. 2010) }\end{array}$ & - \\
\hline & $\begin{array}{l}\text { Sodium Sulfate } \\
\qquad\left(\mathrm{Na}_{2} \mathrm{SO}_{4}\right)\end{array}$ & $\begin{array}{l}95.2 \mathrm{~atm} \\
(2.0 \mathrm{M})\end{array}$ & $\begin{array}{c}2.14 \mu \mathrm{m} / \mathrm{s} \\
\text { (DI water - CTA } \\
\text { flat sheet) }\end{array}$ & $\begin{array}{c}2.7 \mathrm{~g} / \mathrm{m}^{2} . \mathrm{h} \text { at } \\
2.8 \mathrm{MPa}\end{array}$ & Nanofiltration & $\begin{array}{l}\text { Contain scale } \\
\text { precursor ions }\end{array}$ & $\begin{array}{c}\text { Desalination } \\
\text { (Achilli et al. 2010) }\end{array}$ & - \\
\hline & $\begin{array}{l}\text { Potassium Sulfate } \\
\qquad\left(\mathrm{K}_{2} \mathrm{SO}_{4}\right)\end{array}$ & $\begin{array}{l}32.40 \mathrm{~atm} \\
(2.0 \mathrm{M})\end{array}$ & $\begin{array}{c}2.52 \mu \mathrm{m} / \mathrm{s} \\
\text { (DI water - CTA } \\
\text { flat sheet) }\end{array}$ & $\begin{array}{c}3.7 \mathrm{~g} / \mathrm{m}^{2} . \mathrm{h} \text { at } \\
2.8 \mathrm{MPa}\end{array}$ & RO process & $\begin{array}{l}\text { Low water solubility; } \\
\text { High replenishment } \\
\text { cost }\end{array}$ & $\begin{array}{c}\text { Desalination } \\
\text { (Achilli et al. 2010) }\end{array}$ & - \\
\hline & $\begin{array}{l}\text { Magnesium } \\
\text { Sulfate } \\
\left(\mathrm{MgSO}_{4}\right)\end{array}$ & $\begin{array}{l}54.80 \mathrm{~atm} \\
(2.0 \mathrm{M})\end{array}$ & $\begin{array}{c}1.54 \mu \mathrm{m} / \mathrm{s} \\
\text { (DI water - CTA } \\
\text { flat sheet) }\end{array}$ & $\begin{array}{c}1.2 \mathrm{~g} / \mathrm{m}^{2} . h \text { at } \\
2.8 \mathrm{MPa}\end{array}$ & Nanofiltration & $\begin{array}{l}\text { High viscosity; Low } \\
\text { water solubility; } \\
\text { Contain scale } \\
\text { precursor ions }\end{array}$ & $\begin{array}{c}\text { Desalination } \\
\text { (Achilli et al. 2010) }\end{array}$ & - \\
\hline & $\begin{array}{l}\text { Copper Sulfate } \\
\left(\mathrm{CuSO}_{4}\right)\end{array}$ & $\begin{array}{c}29.94 \mathrm{bar} \\
(220,000 \mathrm{ppm})\end{array}$ & $\begin{array}{c}3.57 \mathrm{LMH} \\
\text { (5,050 ppm } \\
\mathrm{NaCl}-\mathrm{CTA} \text { flat } \\
\text { sheet) }\end{array}$ & Not reported & $\begin{array}{c}\text { Metathesis } \\
\text { precipitation with } \\
\text { barium hydroxide } \\
\text { then sulphuric } \\
\text { acid }\end{array}$ & $\begin{array}{l}\text { Low water flux; FO } \\
\text { process severely } \\
\text { affected by } \\
\text { concentration } \\
\text { polarization effect }\end{array}$ & $\begin{array}{c}\text { Brackish water } \\
\text { desalination } \\
\text { (Alnaizy et al. 2013) }\end{array}$ & - \\
\hline
\end{tabular}




\begin{tabular}{|c|c|c|c|c|c|c|c|c|}
\hline $\begin{array}{c}\text { Draw } \\
\text { solution } \\
\text { types }\end{array}$ & Draw solution & $\begin{array}{c}\text { Osmotic } \\
\text { pressure } \\
\text { (concentration) }\end{array}$ & $\begin{array}{c}\text { Water flux } \\
\text { (Feed solution } \\
\text { - membrane) } \\
\end{array}$ & $\begin{array}{l}\text { Reverse salt } \\
\quad \text { flux }\end{array}$ & $\begin{array}{l}\text { Recovery } \\
\text { process(es) }\end{array}$ & Drawbacks & $\begin{array}{l}\text { Example(s) of } \\
\text { application }\end{array}$ & $\begin{array}{l}\text { Commercial } \\
\text { status }\end{array}$ \\
\hline \multirow{5}{*}{$\begin{array}{l}\text { Inorganic } \\
\text { salts }\end{array}$} & $\begin{array}{l}\text { Sodium Nitrate } \\
\qquad\left(\mathrm{NaNO}_{3}\right)\end{array}$ & $\begin{array}{l}81.10 \mathrm{~atm} \\
(2.0 \mathrm{M})\end{array}$ & $\begin{array}{c}5.706 \mu \mathrm{m} / \mathrm{s} \\
\text { (DI water - CA } \\
\text { embedded in } \\
\text { polyester } \\
\text { woven mesh) } \\
\end{array}$ & $\begin{array}{c}0.278 \\
\mathrm{mmoles} / \mathrm{m}^{2} . \mathrm{s}\end{array}$ & Direct application & $\begin{array}{l}\text { High reverse salt } \\
\text { diffusion }\end{array}$ & $\begin{array}{l}\text { Direct fertigation } \\
\text { (Phuntsho et al. 2011) }\end{array}$ & - \\
\hline & $\begin{array}{c}\text { Potassium } \\
\text { Nitrate }\left(\mathrm{KNO}_{3}\right)\end{array}$ & $\begin{array}{l}64.90 \mathrm{~atm} \\
(2.0 \mathrm{M})\end{array}$ & $\begin{array}{c}4.429 \mu \mathrm{m} / \mathrm{s} \\
\text { (DI water - CA } \\
\text { embedded in } \\
\text { polyester } \\
\text { woven mesh) }\end{array}$ & $\begin{array}{c}0.486 \\
\mathrm{mmoles} / \mathrm{m}^{2} . \mathrm{s}\end{array}$ & Direct application & $\begin{array}{c}\text { High reverse salt } \\
\text { diffusion; toxic; energy } \\
\text { intensive }\end{array}$ & $\begin{array}{l}\text { Direct fertigation } \\
\text { (Phuntsho et al. 2011) }\end{array}$ & - \\
\hline & $\begin{array}{l}\text { Calcium Nitrate } \\
\left(\mathrm{Ca}\left(\mathrm{NO}_{3}\right)_{2}\right)\end{array}$ & $\begin{array}{c}108.50 \mathrm{~atm} \\
(2.0 \mathrm{M})\end{array}$ & $\begin{array}{c}50.22 \mu \mathrm{m} / \mathrm{s} \\
\text { (DI water - CA } \\
\text { embedded in } \\
\text { polyester } \\
\text { woven mesh) } \\
\end{array}$ & $\begin{array}{c}0.009 \\
\mathrm{mmoles} / \mathrm{m}^{2} . \mathrm{s}\end{array}$ & Direct application & $\begin{array}{l}\text { High replenishment } \\
\text { cost; Poor water } \\
\text { extraction capacity }\end{array}$ & $\begin{array}{c}\text { Direct fertigation } \\
\text { (Phuntsho et al. 2011); } \\
\text { Desalination (Achilli et } \\
\text { al. 2010) }\end{array}$ & - \\
\hline & $\begin{array}{l}\text { Ammonium } \\
\text { Phospate } \\
\left(\mathrm{NH}_{4} \mathrm{H}_{2} \mathrm{PO}_{4}\right)\end{array}$ & $\begin{array}{c}86.30 \mathrm{~atm} \\
(2.0 \mathrm{M})\end{array}$ & $\begin{array}{c}4.349 \mu \mathrm{m} / \mathrm{s} \\
\text { (DI water - CA } \\
\text { embedded in } \\
\text { polyester } \\
\text { woven mesh) } \\
\end{array}$ & $\begin{array}{c}0.069 \\
\mathrm{mmoles} / \mathrm{m}^{2} . \mathrm{s}\end{array}$ & Direct application & Low water flux & $\begin{array}{l}\text { Direct fertigation } \\
\text { (Phuntsho et al. 2011) }\end{array}$ & - \\
\hline & $\begin{array}{l}\text { Diammonium } \\
\text { Phosphate } \\
\left(\left(\mathrm{NH}_{4}\right)_{2} \mathrm{HPO}_{4}\right)\end{array}$ & $\begin{array}{l}95.00 \mathrm{~atm} \\
(2.0 \mathrm{M})\end{array}$ & $\begin{array}{c}3.892 \mu \mathrm{m} / \mathrm{s} \\
\text { (DI water - CA } \\
\text { embedded in } \\
\text { polyester } \\
\text { woven mesh) }\end{array}$ & $\begin{array}{c}0.01 \\
\mathrm{mmoles} / \mathrm{m}^{2} . \mathrm{s}\end{array}$ & Direct application & Low water flux & $\begin{array}{l}\text { Direct fertigation } \\
\text { (Phuntsho et al. 2011) }\end{array}$ & - \\
\hline \multirow{4}{*}{$\begin{array}{c}\text { Novel } \\
\text { synthetic } \\
\text { materials }\end{array}$} & $\begin{array}{l}\text { Polyacrylic acid } \\
\text { MNPs } \\
\text { (PAA MNPs) } \\
\end{array}$ & $\begin{array}{l}\text { Up to } 70 \mathrm{~atm} \\
(0.08 \mathrm{~mol} / \mathrm{L})\end{array}$ & $\begin{array}{c}10 \text { to } 17 \mathrm{LMH} \\
\text { (DI water }-\mathrm{HTI} \\
\text { membrane) }\end{array}$ & Not reported & \multirow{4}{*}{$\begin{array}{l}\text { Magnetic field } \\
\text { separator, FO } \\
\text { process using RO } \\
\text { brine as DS, } \\
\text { Ultrafiltration }\end{array}$} & \multirow{4}{*}{$\begin{array}{l}\text { Slightly drop of water } \\
\text { flux due to } \\
\text { agglomeration of the } \\
\text { MNPs }\end{array}$} & \multirow{3}{*}{$\begin{array}{l}\text { Protein enrichment (Ling } \\
\text { and Chung 2011b); } \\
\text { Water reuse (Ling et al. } \\
\text { 2010); Desalination (Ling } \\
\text { and Chung 2011a) }\end{array}$} & \multirow{4}{*}{ - } \\
\hline & $\begin{array}{l}\text { 2-pyrolidone } \\
\text { based MNPs } \\
\text { (2-Pyrol MNPs) }\end{array}$ & Not reported & $\begin{array}{c}0.5 \text { to } 5 \mathrm{LMH} \\
\text { (DI water }-\mathrm{HTI} \\
\text { membrane) }\end{array}$ & Not reported & & & & \\
\hline & $\begin{array}{l}\text { Triethylene glycol } \\
\text { MNPs } \\
\text { (TREG MNPs) } \\
\end{array}$ & Not reported & $\begin{array}{c}0.5 \text { to } 5 \mathrm{LMH} \\
\text { (DI water }-\mathrm{HTI} \\
\text { membrane) }\end{array}$ & Not reported & & & & \\
\hline & $\begin{array}{l}\text { Polyethylene } \\
\text { glycol diacid } \\
\text { MNPs } \\
\text { (PEG-(COOH) } \\
\text { MNPs) }\end{array}$ & $\begin{array}{l}55 \text { to } 73 \mathrm{~atm} \\
(0.065 \mathrm{~mol} / \mathrm{L})\end{array}$ & $\begin{array}{l}5.3 \text { to } 9.1 \mathrm{LMH} \\
\text { (DI water - CTA } \\
\text { flat sheet) }\end{array}$ & Not reported & & & $\begin{array}{l}\text { No specific application } \\
\quad \text { (Ge et al. 2011) }\end{array}$ & \\
\hline
\end{tabular}




\begin{tabular}{|c|c|c|c|c|c|c|c|c|}
\hline $\begin{array}{l}\text { Draw } \\
\text { solution } \\
\text { types }\end{array}$ & Draw solution & $\begin{array}{c}\text { Osmotic } \\
\text { pressure } \\
\text { (concentration) }\end{array}$ & $\begin{array}{l}\text { Water flux } \\
\text { (Feed solution } \\
\text { - membrane) }\end{array}$ & $\begin{array}{l}\text { Reverse salt } \\
\quad \text { flux }\end{array}$ & $\begin{array}{l}\text { Recovery } \\
\text { process(es) }\end{array}$ & Drawbacks & $\begin{array}{l}\text { Example(s) of } \\
\text { application }\end{array}$ & $\begin{array}{l}\text { Commercial } \\
\text { status }\end{array}$ \\
\hline \multirow{9}{*}{$\begin{array}{c}\text { Novel } \\
\text { synthetic } \\
\text { materials }\end{array}$} & $\begin{array}{l}\text { Nano size dextran } \\
\text { coated Ferric } \\
\text { oxide MNPs } \\
\left(\mathrm{Fe}_{3} \mathrm{O}_{4}\right)\end{array}$ & Not reported & $\begin{array}{c}3.25 \text { to } 4 \mathrm{LMH} \\
\text { (DI water }-\mathrm{HTI} \\
\text { membrane) }\end{array}$ & Not reported & External magnet & $\begin{array}{l}\text { Slightly drop of water } \\
\text { flux due to } \\
\text { agglomeration of the } \\
\text { MNPs }\end{array}$ & $\begin{array}{c}\text { Brackish water } \\
\text { desalination (Bai et al. } \\
\text { 2011) }\end{array}$ & - \\
\hline & $\begin{array}{c}2- \\
\text { methylimidazole } \\
\text { based } \\
\text { compounds with } \\
\text { monovalent and } \\
\text { divalent charges } \\
\end{array}$ & $\begin{array}{l}50 \text { to } 150 \mathrm{bar} \\
(2.0 \mathrm{M})\end{array}$ & $\begin{array}{l}0.1 \text { to } 20 \mathrm{LMH} \\
\text { (DI water - CTA } \\
\text { flat sheet) }\end{array}$ & $\begin{array}{l}5 \text { to } 80 \\
\mathrm{~g} / \mathrm{m}^{2} \cdot h\end{array}$ & $\begin{array}{l}\text { FO-MD integrated } \\
\text { process }\end{array}$ & $\begin{array}{l}\text { High ICP effect when } \\
\text { using compound with } \\
\text { divalent charge; High } \\
\text { reverse solute flux; } \\
\text { High cost }\end{array}$ & $\begin{array}{l}\text { Seawater desalination } \\
\text { (Yen et al. 2010) }\end{array}$ & - \\
\hline & $\begin{array}{l}\text { Polyelectrolytes } \\
\text { of polyacrylic acid } \\
\text { sodium } \\
\text { (PAA-Na) } \\
\end{array}$ & $\begin{array}{l}25-45 \mathrm{~atm} \\
(0.72 \mathrm{mg} / \mathrm{L})\end{array}$ & $\begin{array}{l}13 \text { to } 21 \mathrm{LMH} \\
\text { (DI water - CA } \\
\text { hollow fiber) }\end{array}$ & $0.15 \mathrm{~g} / \mathrm{m}^{2} . \mathrm{h}$ & $\begin{array}{l}\text { FO-MD integrated } \\
\text { process, } \\
\text { Ultrafiltration }\end{array}$ & High viscosity & $\begin{array}{l}\text { Wastewater treatment } \\
\quad \text { (Ge et al. 2012b) }\end{array}$ & - \\
\hline & $\begin{array}{l}\text { Thermo-sensitive } \\
\text { polyelectrolytes }\end{array}$ & $\begin{array}{l}\text { Up to } 88 \text { atm } \\
\text { (14.28 wt\% } \\
\text { polyelectrolytes } \\
\text { with different } \\
\text { sodium acrylate } \\
\text { content) }\end{array}$ & $\begin{array}{l}0.05 \text { to } 0.75 \\
\text { LMH (DI water- } \\
\text { HTI membrane) }\end{array}$ & Not reported & Hot ultrafiltration & Poor water flux & $\begin{array}{l}\text { Desalination (Ou et al. } \\
\text { 2013) }\end{array}$ & - \\
\hline & $\begin{array}{l}\text { Polymer } \\
\text { hydrogels }\end{array}$ & 2.7 MPa & $\begin{array}{c}0.55 \text { to } 1.1 \mathrm{LMH} \\
(2000 \mathrm{ppm} \mathrm{NaCl} \\
-\mathrm{HTI} \\
\text { membrane) } \\
\end{array}$ & Not reported & $\begin{array}{l}\text { Direct application, } \\
\text { Heating, Pressure } \\
\text { Stimuli }\end{array}$ & $\begin{array}{l}\text { Energy intensive; Poor } \\
\text { water flux }\end{array}$ & $\begin{array}{l}\text { Seawater desalination } \\
\text { (Li et al. 2011b; Li et al. } \\
\text { 2013) }\end{array}$ & - \\
\hline & Acyl-TAEA & N.A. & N.A. & N.A. & $\begin{array}{c}\text { High } \\
\text { temperatures }\end{array}$ & Poor water flux & $\begin{array}{l}\text { Seawater desalination } \\
\text { (Noh et al. 2012) }\end{array}$ & - \\
\hline & $\begin{array}{l}\text { Micelles close to } \\
\text { the Kraft point }\end{array}$ & $95.1 \mathrm{~Pa}$ & $\begin{array}{l}4.73 \text { to } 16.14 \\
\quad \text { LMH } \\
\text { (n.a.) }\end{array}$ & Not reported & $\begin{array}{l}\text { Temperature } \\
\text { swing with low } \\
\text { grade heat and } \\
\text { crystallisation } \\
\end{array}$ & Low diffusivity & $\begin{array}{l}\text { Seawater desalination } \\
\text { (Gadelha and Hankins } \\
\text { 2011) }\end{array}$ & - \\
\hline & Dendrimers & $\begin{array}{c}330 \mathrm{psi} \\
(22.5 \mathrm{~atm}) \\
(20 \mathrm{wt} . \%)\end{array}$ & Not tested & Not tested & $\begin{array}{l}\text { Wide range of } \mathrm{pH} \\
\quad \text { value and } \\
\text { ultrafiltration }\end{array}$ & \multirow{2}{*}{ Not feasible } & \multirow{2}{*}{$\begin{array}{l}\text { Dewatering Reverse } \\
\text { Osmosis concentrate } \\
\text { (Adham et al. 2009) }\end{array}$} & - \\
\hline & Albumin & $\begin{array}{c}7 \text { psi } \\
\text { (30 wt. \%) }\end{array}$ & Not tested & Not tested & $\begin{array}{l}\text { Denatured and } \\
\text { solidified upon } \\
\text { heating }\end{array}$ & & & - \\
\hline
\end{tabular}




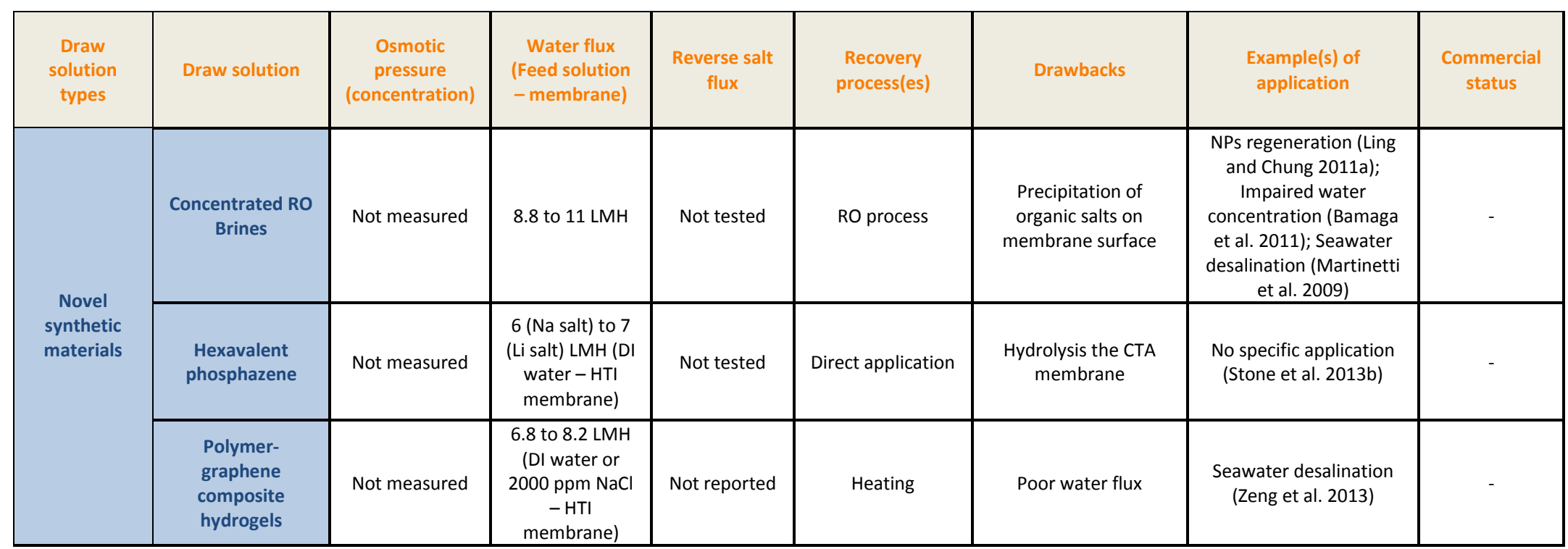

* The osmotic pressure, water flux and reverse salt flux data were obtained from studies highlighted in bold. 
In order to develop a continuous FO process including the DS recovery process, McGinnis (2002) introduced the use of potassium nitrate $\left(\mathrm{KNO}_{3}\right)$ and $\mathrm{SO}_{2}$ as DS for desalination application. This concept consisted of a two-stage FO process (Figure 5.2): Hot seawater and saturated $\mathrm{KNO}_{3}$ solution were used as feed and draw solution respectively in the first unit while the diluted $\mathrm{KNO}_{3}$ from the first $\mathrm{FO}$ unit is used as feed solution in the second unit and reconcentrated by a saturated $\mathrm{SO}_{2}$ solution, used as DS. The $\mathrm{SO}_{2}$ is removed by standard means such as heating. However, performance data are not available till now.

$\mathrm{SO}_{2}$ was also suggested as potential DS in the early 1960's. Batchelder (1965) was the first to test $\mathrm{SO}_{2}$ for desalination application. In the patent, the separation of $\mathrm{SO}_{2}$ from the product water was made by heating or air stripping. However, although a positive water flux was observed in the experiments; neither the osmotic pressure, nor the water flux and salt rejection were reported in the patent. In the same year, Glew (1965) proposed a mixture of water and $\mathrm{SO}_{2}$ or aliphatic alcohol as DS. The purpose of adding $\mathrm{SO}_{2}$ or aliphatic alcohol to water is to increase the osmotic pressure of the resulting solution. In this study, the recovery of $\mathrm{SO}_{2}$ was proposed by means of distillation.

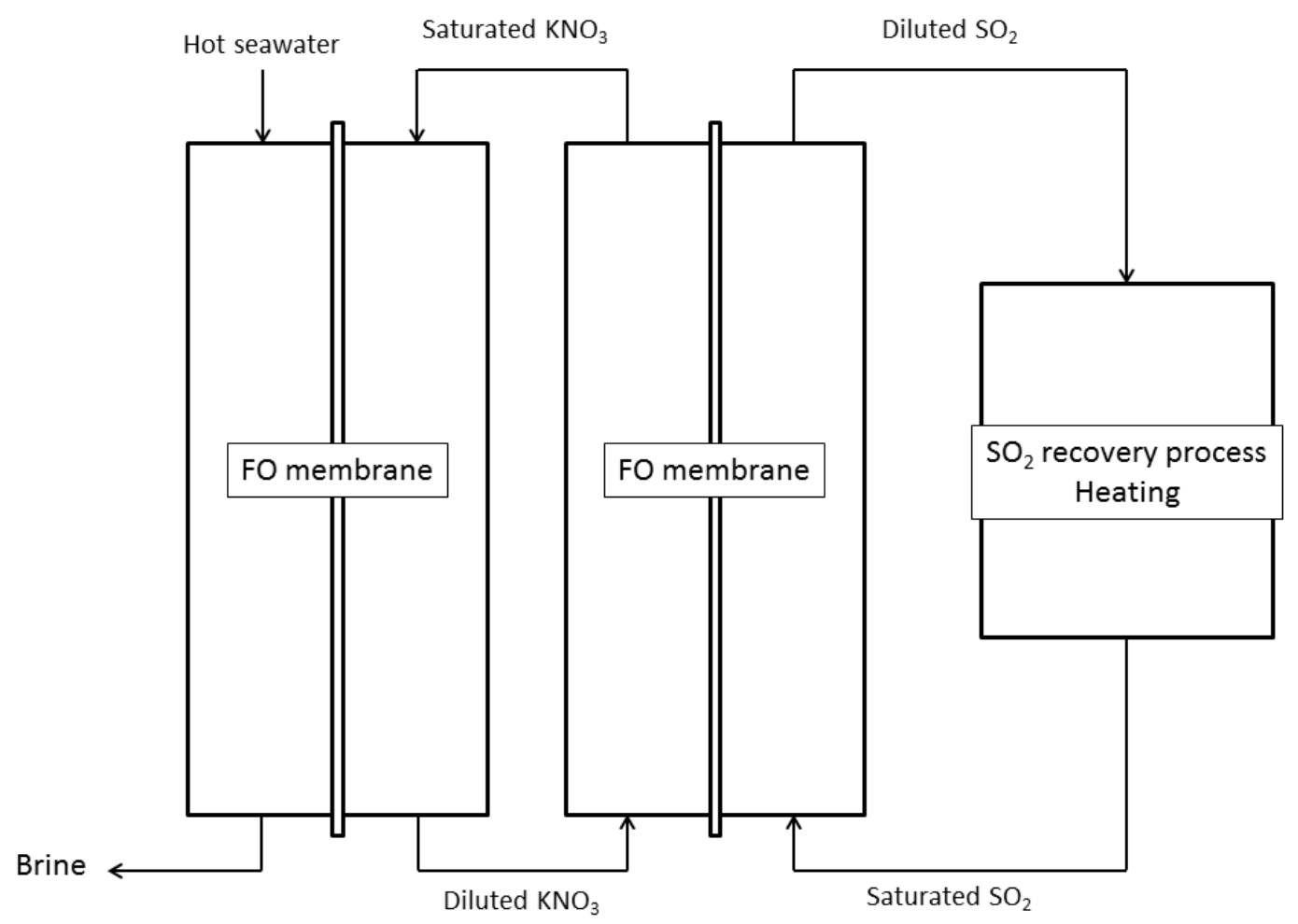

Figure 5.2 Schematic representation of the two-stage FO process for desalination 


\subsubsection{Organic Compounds}

Over the past decades, organic compounds have been tested as DS for various applications, including mainly food production (Beaudry and Lampi 1990; Wrolstad et al. 1993; Herron et al. 1994; Petrotos et al. 1998) and seawater desalination (Kravath and Davis 1975; Kessler and Moody 1976; Stache 1989; Ng and Tang 2006). Although organic compounds are generally non-charged compounds, they display high solubility in water and can generate high osmotic pressure (Ng and Tang 2006).

\subsubsection{Nutrient Compounds}

The study of nutrient compounds as DS originally focused on desalination applications for drinking water production, where the diluted DS can be used directly, excluded the need for its recovery. Kravath and Davis (1975) were the first to explore glucose as a DS for emergency water supply in lifeboats. The diluted glucose solution, present in the final product, was edible and did not require further treatment. Following the work of Kravath and Davis, Kessler and Moody (1976) investigated the combination of glucose and fructose as DS. Better performance was achieved in comparison to pure glucose solution. Glycine was also suggested as DS for the same application as it can produce a higher osmotic pressure than fructose; however, it can be toxic for human consumption at high concentration (Stache 1989). In the work, Stache (1989) demonstrated that, to be efficient, the fructose content must be at least $90 \%(\mathrm{w} / \mathrm{w})$ to deliver an appropriate water flux. He also showed that below $74 \%$ (w/w), the water flux may be reversed from the draw solution to the saline feed solution. Later, Yaeli (1992) developed a hybrid system combining FO process and low pressure RO process for the recovery of glucose.

The use of nutrient compounds as DS is still attracting attention recently (Wallace et al. 2008; Su et al. 2012; Yong et al. 2012) and HTI has recently developed and manufactured the first commercial hydration bags using FO technology where sugar or beverage powder is used as DS (HTI 2010). In their recent work, Su et al. (2012) found that sucrose was more effective than $\mathrm{MgCl}_{2}$ as DS. This was explained by the large molecular size of sucrose resulting in a very low reverse draw solution flux; producing an almost constant osmotic gradient across the membrane during the time of the experiments.

\subsubsection{Organic Salts}

Organic salts are defined as the mixture of an organic anion and an organic or inorganic cation. In a recent study, Bowden et al. (2012) tested a series of organic salts as potential DS in an osmotic membrane bioreactor (OMBR) for wastewater treatment as shown in Figure 5.3. After an initial screening based on ten organic salts, they 
selected four potential DS for further tests in OMBR, RO reconcentration and biodegradation. The advantage of using biodegradable organic salts is to moderate the accumulation of DS inside the bioreactor. They found that the organic salts with shorter carbon chain displayed better performance that the ones with longer carbon chains when they have the same cation species at the same osmotic pressure. This is because the organic salts with shorter carbon chain have higher diffusion coefficient and thus generate less ICP effects. Compared to pure inorganic salts (i.e. $\mathrm{NaCl}$ and $\mathrm{MgCl}_{2}$ ), organic salts were proved to display higher salt rejection in the RO reconcentration process but the produced water fluxes were much lower under the same conditions.

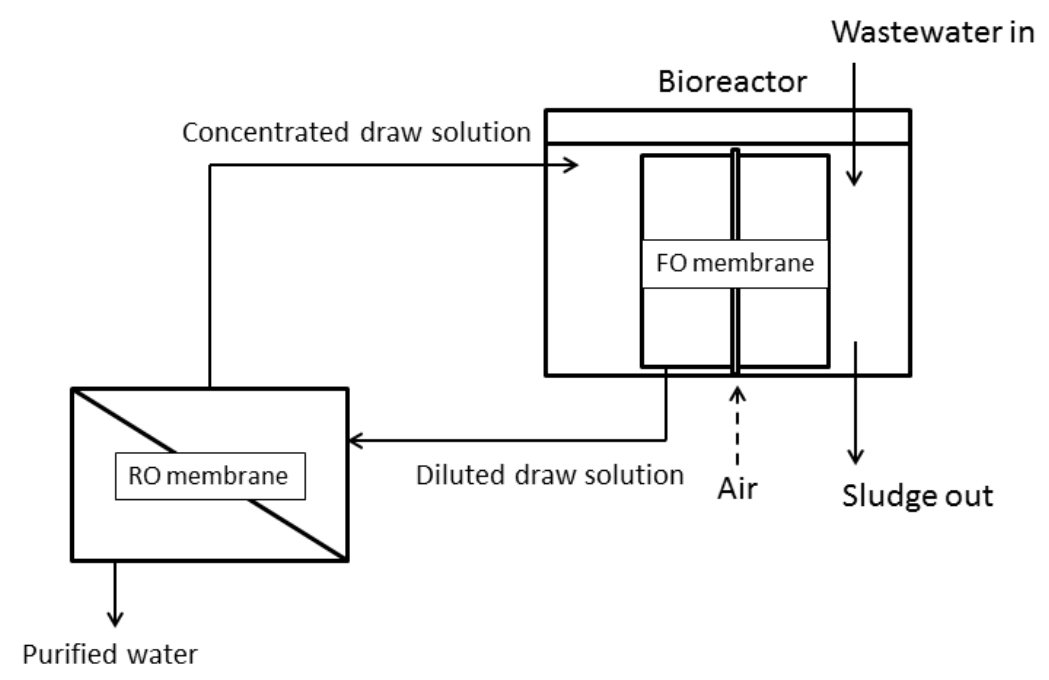

Figure 5.3 Schematic of the OMBR process (adapted from Bowden et al. 2012)

\subsubsection{Switchable Polarity Solvents (SPS)}

In a recent work carried out by the Idaho National Laboratory (Stone et al. 2013a), switchable polarity solvents (SPS) (Jessop et al. 2005, 2011, 2012) have been tested as potential novel DS for the industrial purification of water. SPS are mixtures of $\mathrm{CO}_{2}$, water and tertiary amines. Their principal characteristic is their ability to switch from polar to non-polar form in the presence or not of $\mathrm{CO}_{2}$, as shown in Figure 5.4.

$$
\underset{\text { Non-polar }}{\mathrm{NR}_{3}+\mathrm{CO}_{2}+\mathrm{H}_{2} \mathrm{O} \leftrightarrow \underset{\text { Polar }}{\mathrm{HNR}_{3}{ }^{+}+\mathrm{HCO}_{3}^{-}}}
$$

Figure 5.4 General example of an SPS from its non-polar to its polar form

This unique characteristic makes their recovery very straightforward, simply by adding 1 atm of air or $\mathrm{N}_{2}$ with mild heating to form hydrophobic SPS. Then, the hydrophobic SPS can be regenerated and recycled to hydrophilic SPS by adding $1 \mathrm{~atm}$ of $\mathrm{CO}_{2}$. Further 
treatment can be done to remove the trace amount of hydrophobic SPS from the produced water with RO process; as depicted in Figure 5.5.

Regarding FO performance, these DS were able to generate a very high osmotic pressure, up to $325 \mathrm{~atm}$, and produce a reasonable water flux, up to $35 \mathrm{LMH}$, depending on the concentration of $\mathrm{NaCl}$ in the feed solution. Poor RSF was observed at $\mathrm{NaCl}$ concentration below $0.75 \mathrm{~mol} / \mathrm{kg}$. At higher concentration, RSF was also relatively low (e.g., up to $1.8 \mathrm{wt} \%$ of the initial DS concentration). However, under the tested experimental conditions, authors found that SPS degraded the FO membrane.

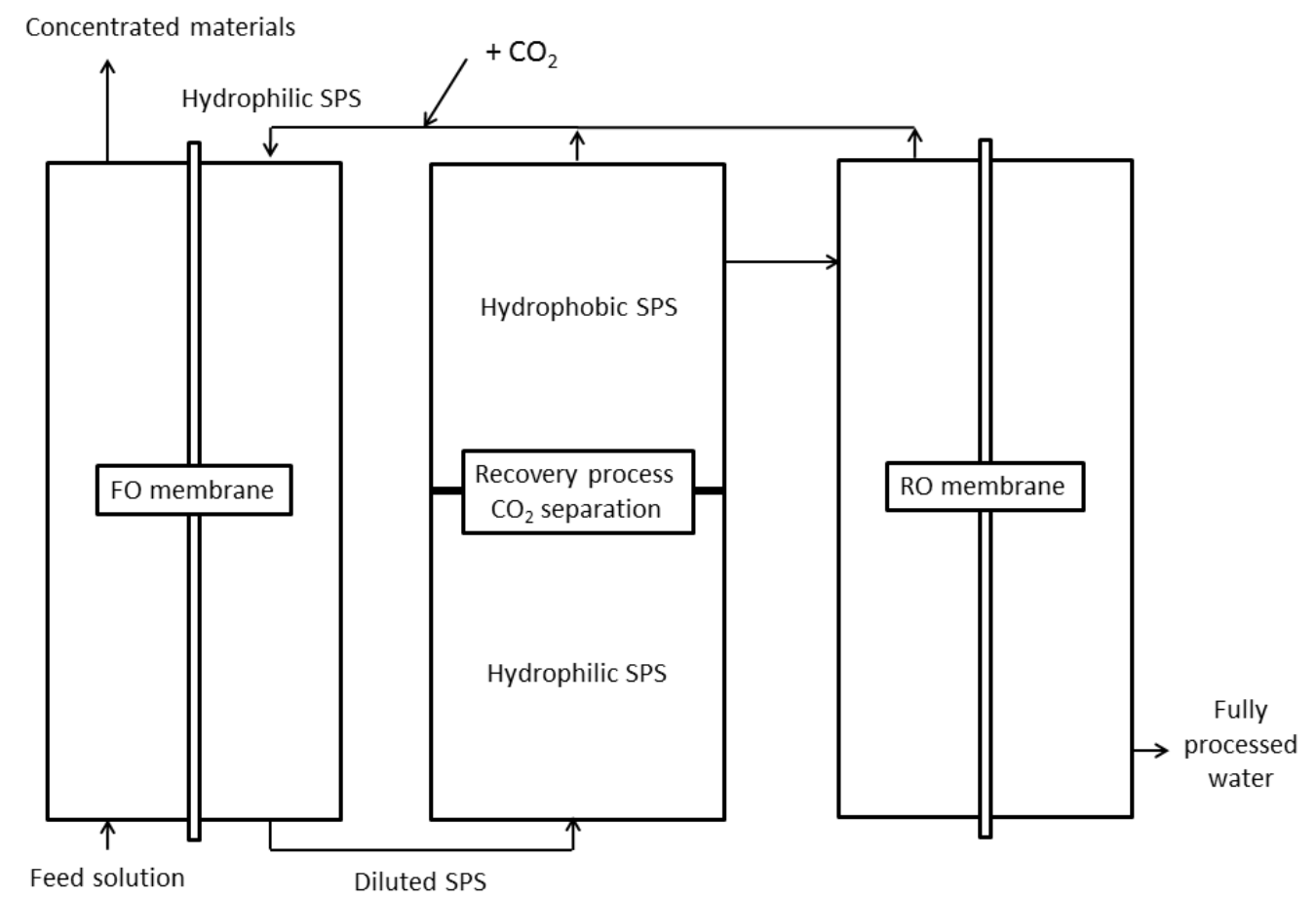

Figure 5.5 Schematic representation of the proposed SPS FO process

\subsubsection{Inorganic Compounds}

The vast majority of FO studies have tested inorganic-based compounds as DS, and they are still widely used nowadays. Inorganic-based compounds are mainly composed of electrolyte solutions although some non-electrolyte solutions could be also encountered. The use of inorganic compounds as DS has attracted many researchers since they can generally produce reasonably high water fluxes and can be easily recovered by RO (Achilli et al. 2010) or NF (Tan and Ng 2010) or used directly without further treatment (e.g. for fertigation) (Phuntsho et al. 2011). 
The study of inorganic compounds as DS started in 1970 when Hough tested a variety of acids such as manganic acid, valeric acid and glucuronic acid and a range of inorganic salts such as calcium, potassium, sodium, barium and cesium salts as DS for desalination application. In the patent, their temperature-sensitive solubility or reactions with sulphuric acid or carbon dioxide to extract water by precipitating these DS were utilised. Later, Frank (1972) investigated the use of aluminium sulphate as DS for seawater desalination and their recovery by precipitation with calcium sulphate. The major drawback of these DS was the excessive use of chemicals for their recovery.

The most recent and comprehensive studies of inorganic compounds as DS were proformed by Phuntsho et al. (2011), Achilli et al. (2010) and Tan and Ng (2010). In their study, Achilli and co-workers tested and compared 14 inorganic compounds as DS for potential application in desalination. These DS were selected from among 500 inorganic compounds due to their higher water solubility and osmotic pressure and lower cost and toxicity. The performance of the selected DS was evaluated in terms of water fluxes and RSF through the FO process and their recovery were tested using a RO process design software. DS containing larger hydrated anions (i.e. $\mathrm{MgSO}_{4}, \mathrm{KHCO}_{3}$, $\mathrm{NaHCO}_{3}, \mathrm{Na}_{2} \mathrm{SO}_{4},\left(\mathrm{NH}_{4}\right)_{2} \mathrm{SO}_{4}$ and $\left.\mathrm{K}_{2} \mathrm{SO}_{4}\right)$ showed the lowest RSF. However, based on the overall performance (i.e. FO performance and recovery test), none of the 14 tested DS outperformed the others. Tan and Ng (2010) investigated a novel hybrid system combining FO and NF for seawater desalination (Figure 5.6); seven potential DS were tested at laboratory scale, including six inorganic compounds (i.e. $\mathrm{NaCl}, \mathrm{KCl}, \mathrm{MgCl}_{2}$, $\mathrm{CaCl}_{2}, \mathrm{MgSO}_{4}, \mathrm{Na}_{2} \mathrm{SO}_{4}$ ). Their results showed that $\mathrm{KCl}$ and $\mathrm{NaCl}$ exhibit the highest water flux of more than $25 \mathrm{LMH}$ while $\mathrm{MgCl}_{2}$ and $\mathrm{CaCl}_{2}$ produced the highest osmotic pressure due to their high solubility in water. Experiments also showed that all DS displayed high solute rejection (i.e. above $99 \%$ ). Only $\mathrm{MgCl}_{2}, \mathrm{MgSO}_{4}$ and $\mathrm{Na}_{2} \mathrm{SO}_{4}$ were tested on the hybrid $\mathrm{FO} / \mathrm{NF}$ system and experiments showed that the system was able to produce high quality water when using $\mathrm{MgSO}_{4}$ and $\mathrm{Na}_{2} \mathrm{SO}_{4}$ as DS. Finally, in a recent study by Phuntsho et al. (2011), fertilisers were proposed as DS to extract water from brackish sources for direct irrigation. The diluted fertilisers can be applied directly without any further treatment as a source of nutrient for irrigation. In their study, They investigated nine commonly used fertilisers as DS and assessed their performance in terms of pure water flux and reverse solute flux. $\mathrm{KCl}, \mathrm{NaNO}_{3}$ and $\mathrm{KNO}_{3}$ exhibited the highest water fluxes while $\mathrm{NH}_{4} \mathrm{H}_{2} \mathrm{PO}_{4}$ and $\left(\mathrm{NH}_{4}\right)_{2} \mathrm{HPO}_{4}$ showed the lowest. The ammonium compounds of phosphate and sulphate (i.e. $\mathrm{NH}_{4} \mathrm{H}_{2} \mathrm{PO}_{4},\left(\mathrm{NH}_{4}\right)_{2} \mathrm{HPO}_{4}$, $\left(\mathrm{NH}_{4}\right)_{2} \mathrm{SO}_{4}$ and $\left.\mathrm{Ca}\left(\mathrm{NO}_{3}\right)_{2}\right)$ had the lowest reverse solute fluxes since they possess ionic species with large hydrated diameter. Based on the results, they concluded that the choice of DS for fertigation mainly depends on the compatibility with the FO membrane as well as the plant nutrient requirements. In a more recent study (Phuntsho et al. 2012), they tested blended fertilisers as DS but the obtained osmotic pressures and water fluxes were lower than the sum of the two individual DS. 


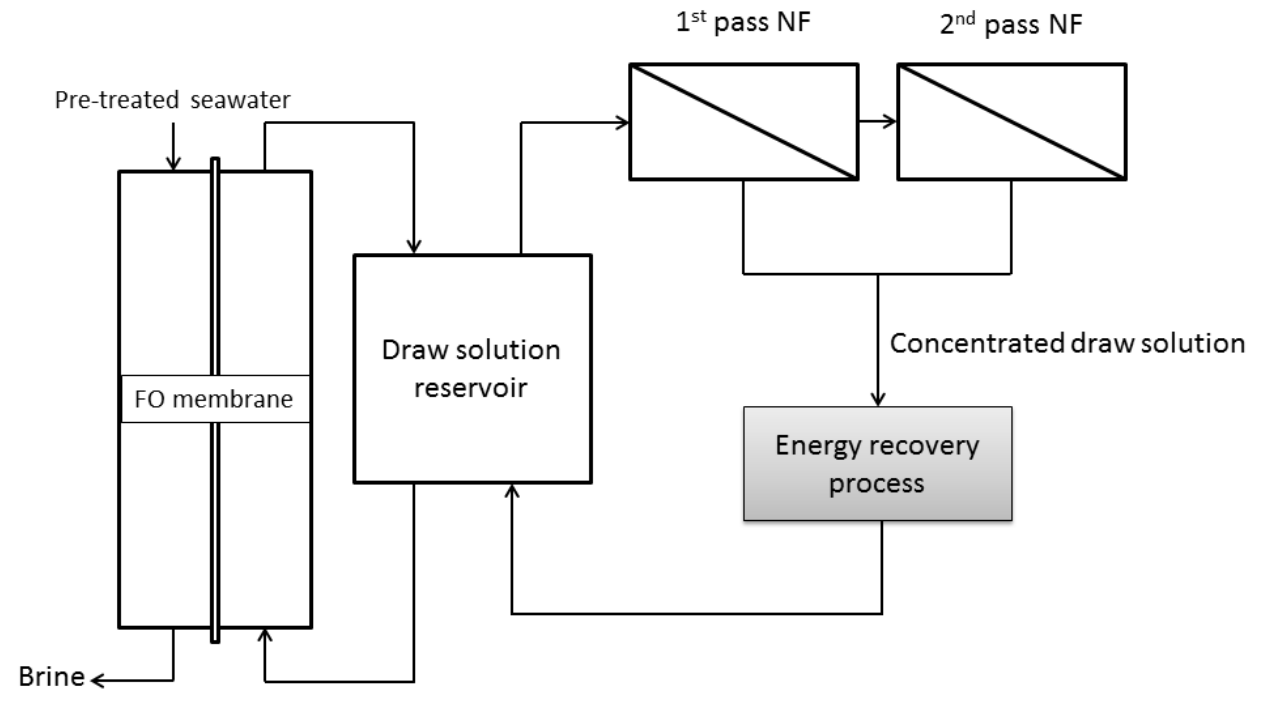

Figure 5.6 Schematic representation of the proposed FO-NF process for seawater desalination (adapted from Tan and Ng, 2010)

Sodium chloride (i.e. $\mathrm{NaCl}$ ) has been widely used as DS in a variety of applications, including food production (Petrotos et al. 1998; Garcia-Castello et al. 2009) and water and wastewater treatment (York et al. 1999; Holloway 2006; Achilli et al. 2009; Martinetti et al. 2009). The advantages of $\mathrm{NaCl}$ are that it is abundant on earth, making seawater a natural and inexpensive source of DS, it is relatively easy to reconcentrate with RO process and it has high water solubility and osmotic pressure. Besides, the thermodynamic properties of $\mathrm{NaCl}$ have been widely investigated, making it easier to study.

Finally, a recent study by Alnaizy et al. (2013) investigated the use of copper sulfate $\left(\mathrm{CuSO}_{4}\right)$ as draw solution for desalination applications. They found that $\mathrm{CuSO}_{4}$ cannot produce enough osmotic pressure to desalinate seawater but could be a viable candidate for brackish water desalination. The proposed recovery method consisted in metathesis precipitation reaction with barium hydroxide followed by reaction with sulphuric acid.

\subsubsection{Novel Synthetic Compounds}

\subsubsection{Hydrophilic Magnetic Nanoparticles (MNPs)}

Hydrophilic magnetic nanoparticles (MNPs) have gained growing interest in the past few years as promising DS for FO process (Adham et al. 2009; Ling et al. 2010; Warne 2010; Bai et al. 2011; Ge et al. 2011; Ling and Chung 2011b; Ling and Chung 
2011a; Ling et al. 2011; Ling and Chung 2012). Warne et al. (2010) were the first to introduce the concept of using MNPs as DS. In the patent, they synthesised MNPs via multi-step reactions and their regeneration process consisted of an external magnetic field. However, very few data were provided in their study to demonstrate the feasibility of this novel class of DS. The same year, Chung and co-workers successfully applied MNPs as DS for FO processes (Ling et al. 2010; Ge et al. 2011; Ling and Chung 2011b; Ling and Chung 2011a; Ling et al. 2011). In their different studies, they synthesised various MNPs via one-pot reactions and capped them with different surface coatings. Four different MNPs were investigated as potential DS, including: the polyacrylic acid magnetic nanoparticles (PAA MNPs), the 2-Pyrrolidonemagnetic nanoparticles (2-Pyrol MNPs), the triethylene glycol magnetic nanoparticles (TREG MNPs) and the polyethylene glycol diacid (PEG-(COOH $)_{2}$ MNPs). Moderate performances were achieved in terms of water fluxes (i.e. up to $18 \mathrm{LMH}$ ) (Ling et al. 2010; Ge et al. 2011). However, performance of MNPs in terms of reverse fluxes was very high compared to inorganic compounds such as $\mathrm{NaCl}$ and $\mathrm{MgCl}_{2}$ due to their large particle sizes.

The recovery of MNPs from the produced water was proposed by various methods including magnetic field (Ling et al. 2010; Bai et al. 2011; Ge et al. 2011) and low pressure membrane processes such as ultrafiltration (UF) (Ling and Chung 2011a). However, it was observed that under high-strength magnetic field, MNPs have a strong tendency to agglomerate which decreases significantly the performance of the FO process. Ultrasonication was used to overcome this issue but was not able to effectively restore MNPs without changing their magnetic properties (Ling and Chung 2011a). Therefore, UF was found to be more effective in recycling the MNPs. Thermoresponsive MNPs were also synthesised (Ling et al. 2011) and their recovery process only consisted in temperature stimulus. In fact, by heating the diluted MNPs solution above the critical temperature of the thermo-responsive MNPs, the MNPs will agglomerate spontaneously and can then be separated from the produced water by UF or low-strength magnetic field.

The dextran-coated $\mathrm{Fe}_{3} \mathrm{O}_{4} \mathrm{MNPs}$ as a new draw solute was successfully synthesized and used for the FO desalination process (Bai et al. 2011). $\mathrm{Fe}_{3} \mathrm{O}_{4}$ MNPs can be separated from water by an external magnet field simply and it has good water solubility because it is coated with highly soluble organic substances. Dextran, a natural polysaccharide with a variety of molecular weight, is highly water soluble, which can generate as high osmotic pressure as inorganic salts (Cath et al. 2006). Therefore, $\mathrm{Fe}_{3} \mathrm{O}_{4}$ MNPs can be a candidature for the FO process as DS due to its strong magnetism resulting in easy separation with high osmotic pressure. The performance of the dextran coated $\mathrm{Fe}_{3} \mathrm{O}_{4}$ MNPs were investigated in terms of water flux in a lab-scale FO system for water reuse and desalination. Thus, two kinds of the dextran coated $\mathrm{Fe}_{3} \mathrm{O}_{4} \mathrm{MNPs}$ containing different concentration of the dextran were demonstrated as draw solutes. Experimental results showed that the water flux of the dextran coated $\mathrm{Fe}_{3} \mathrm{O}_{4} \mathrm{MNPs}$ containing higher dextran as DS was higher than that of the dextran coated $\mathrm{Fe}_{3} \mathrm{O}_{4} \mathrm{MNPs}$ 
containing lower dextran as DS. This result indicated that the dextran coated $\mathrm{Fe}_{3} \mathrm{O}_{4}$ MNPs containing higher dextran can generate higher osmotic pressure leading to the higher driving force through the membrane. It was proved that the dextran coated $\mathrm{Fe}_{3} \mathrm{O}_{4}$ MNPs DS can draw clean water from the saline water and can be easily separated from the diluted DS by an external magnet depending on its super-paramagnetism.

Most recently, surface-ionized MNPs have been investigated as potential DS in FO (Ling and Chung 2012). These MNPs showed improved FO performance after the surface ionization and could be recovered via an integrated process comprising an electric field and a NF system. More details on MNPs are given in another chapter.

\subsubsection{2-methylimidazole Based Compounds}

Yen et al. (2010) synthesised novel organic compounds, both neutral and charged 2-methylimidazole based compounds and investigated their application for the FO process. The synthesised compounds were able to extract water from seawater and recovered by an integrated FO-MD (i.e. membrane distillation) process. However, this novel class of DS exhibited high reverse solute flux and the cost of synthesis remains very high.

\subsubsection{Polyelectrolytes of Polyacrylic Acid Sodium (PAA-Na)}

Recently, Ge and co-workers developed polyelectrolytes of polyacrylic acid sodium (PAA-Na) and tested its potential as DS (Ge et al. 2012a; Ge et al. 2012b). Their high solubility, flexible configuration and easy recovery via UF process make them suitable DS candidate for FO process. Experimental results showed that these new DS can achieve high water fluxes with very low reverse solute fluxes. A high salt rejection was also obtained when recycling PAA-Na with UF process. The recycled DS showed repeatable performance and indicated no aggregation problems in comparison with MNPs. However, at high concentration, PAA-Na solution showed a very high viscosity, limited its application at ambient conditions. To overcome this issue, a combined $\mathrm{FO} / \mathrm{MD}$ process has been applied to increase the performance of this DS since MD works at high temperature (Ge et al. 2012b). In this recent study, PAA-Na salts were used as DS in an FO-MD hybrid system to recover water from a model wastewater containing acid dye as shown in Figure 5.7.

More recently, new thermo-sensitive polyelectrolytes were evaluated as DS for the FO process (Ou et al. 2013). Polyelectrolytes were synthesised by copolymerization of $\mathrm{N}$-isopropylacrylamide with different amounts of sodium acrylate (SA) and thus nine different polyelectrolytes were prepared and tested in a lab-scale FO process as DS; these prepared draw solutes were thermo-sensitive and water soluble. In this study, hot ultrafiltration (HUF) process was used as a low-energy technique to separate the water from the polyelectrolyte draw solutions which was operated at $45^{\circ} \mathrm{C}$ and at 2 bar. 
Among nine prepared draw solutes, 4\% poly N-isopropylacrylamide (PNIPAM)-SA solution showed the best performance in terms of the water flux (i.e. $0.347 \mathrm{LMH}$ ) in the FO process and water recovery fraction was around 65\% in the HUF process. Although the produced water flux was relatively low, the ease of water recovery and reused would prove that new thermo-sensitive polyelectrolytes used in this study would have a potential of a good candidate for the FO process.

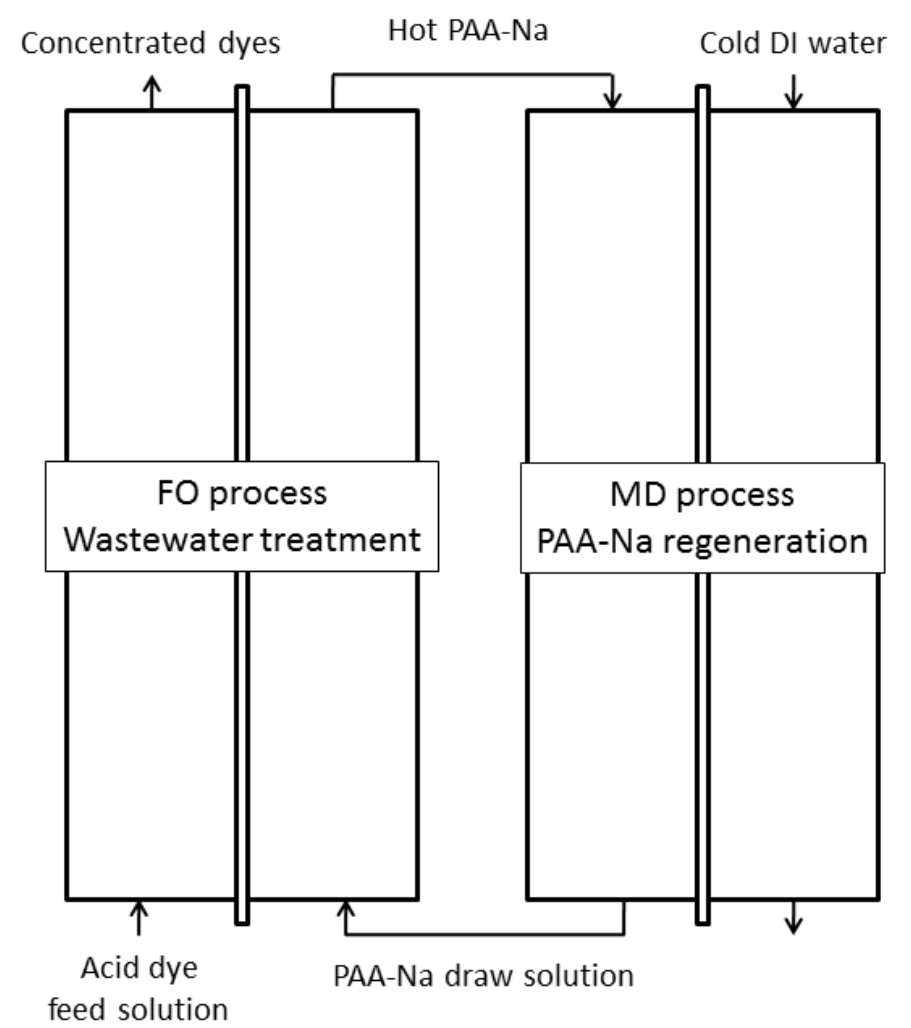

Figure 5.7 Schematic representation of the proposed FO-MD process for dye wastewater treatment (adapted from Ge et al. 2012b)

\subsubsection{Stimuli-Responsive Polymer Hydrogels}

Recently, Li et al. (2011a) have developed a new class of DS, the stimuliresponsive polymer hydrogels for desalination application. Polymer hydrogels are threedimensional networks of polymer chains linked by either physical or chemical bonds. They are able to extract and release water in response to environmental stimuli (e.g. temperature, pressure or light). One interesting response to these stimuli is the change from hydrophilic to hydrophobic which make the polymer hydrogels to release water. This unique property is also advantageous for their recovery which becomes very easy 
and at low energy cost (compared to membrane or thermal processes). In a more recent study (Li et al. 2011c), polymer hydrogels were combined with light-absorbing carbon particles to enhance their heating and dewatering. In both studies, the polymer hydrogels showed high osmotic pressure of about $2.7 \mathrm{MPa}$ at $27^{\circ} \mathrm{C}$. However, FO performances were very poor at room temperature, with water fluxes ranging from 0.55 to $1.1 \mathrm{LMH}$ only. Increasing the temperature to $50{ }^{\circ} \mathrm{C}$ did improve the performance but required a high hydraulic pressure of 30 bars during the dewatering process.

In order to enhance the swelling ratio of the polymer hydrogel used as DS for the FO process, carbon filler particles were incorporated in the polymer hydrogel (Li et al. 2013). The poly (sodium acrylate) hydrogel was selected in this study due to its good performances in terms of high swelling ratio and water flux as compared with the nonionic poly ( $\mathrm{N}$-isopropylacrylamide) or the copolymers of sodium acrylate and $\mathrm{N}$ isopropylacrylamide. Although the addition of carbon particles increased the swelling pressure of hydrogels and led to enhanced water flux, excessive carbon fillers also contributed to a dramatic decrease in the swelling ratio of the composite hydrogels resulting in a lower flux in the FO process. In addition, the effect of hydrogel particle size on the FO process was examined. Smaller composite polymer hydrogel particles (ranging from $100 \mu \mathrm{m}$ to $200 \mu \mathrm{m}$ as draw agents) induced higher water fluxes in FO process as compared with those with larger particle sizes (500-700 $\mu \mathrm{m}$ as draw agents). Furthermore, increased osmotic pressure of the feed solution attributed to the decreasing water flux in the FO process. More details on these DS are given in another chapter.

\subsubsection{Acyl-TAEA}

Lee and co-workers (Noh et al. 2012) recently developed thermo-sensitive DS based on N,N',N' - triacylated tris (2-aminoethyl) amine (acyl-TAEA) derivatives. These new types of DS were able to extract water from saline water when temperatures were lower than the phase separation temperature while the recovery of DS required higher temperature. However, the FO performance of this novel DS is still poor and need further investigation.

\subsubsection{Micellar Solutions}

Gadêlha and Hankins (2011) recently introduced the concept of using micellar solutions as DS in FO process. Micellar solutions have interesting characteristics; they exhibit almost constant osmotic pressure above the critical micelle concentration which enables many dilutions prior to regeneration. Their solubility is highly temperature sensitive around the Krafft point (i.e. critical minimum temperature under which micelles cannot form). This property makes their regeneration very straightforward by simply applying small temperature fluctuations by using low-grade heating and cooling. They can also act as monomeric concentration buffers which attenuate the ICP effects within the membrane. 


\subsubsection{Dendrimers}

Dendrimers are symmetrical globular or spheroid nanostructures, engineered to carry molecules. They consisted of a highly branched tree-like structure linked to a central core via covalent bonds. They were suggested and tested as DS for dewatering RO concentrate in a recent study by Adham et al. (2009). These macromolecules were able to produce a very high osmotic pressure, up to 330 psi (i.e. $22.5 \mathrm{~atm}$ ), which is much higher than the osmotic pressure of RO concentrate. Another advantage is that they can be easily regenerated by low-pressure membrane processes such as UF. However, FO performances, in terms of water fluxes and reverse fluxes, were not assessed in this study.

\subsubsection{Concentrated RO Brines}

Some recent studies have investigated the use of RO brines as DS in order to solve the issue of its disposal. Ling and Chung (2011b) proposed a new FO system for protein enrichment in which MNPs are used as DS in the first FO process to concentrate proteins and RO brine is used as DS in the second FO process to reconcentrate the MNPs. Bamaga et al. (2011) also investigated the use of RO brine as DS in a hybrid $\mathrm{FO} / \mathrm{RO} / \mathrm{FO}$ system (Figure 5.8) where the first FO process is used as a pre-treatment for $\mathrm{RO}$ desalination to minimise scaling risk. The second FO process, using RO brine as DS, is used to concentrate the impaired water to minimise its volume for post-treatments. The combined FO/RO system has recently been successfully designed and commercialised by Modern Water (Thompson and Nicoll 2011) and the first commercialised FO desalination plant has been opened in Oman on the Arabian Sea.

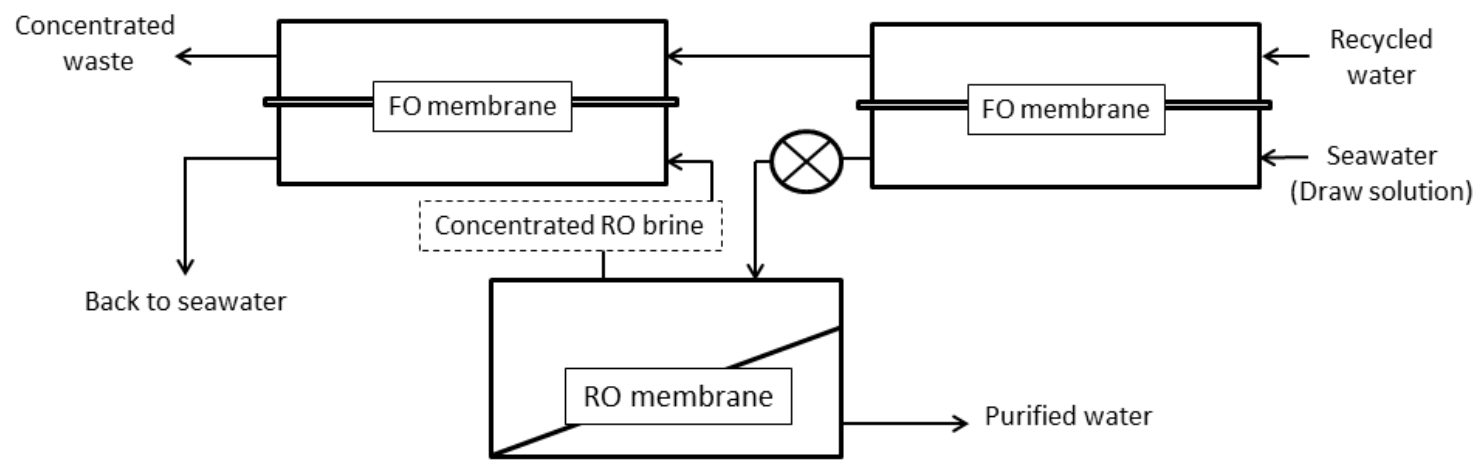

Figure 5.8 Schematic of the hybrid FO/RO system (adapted from Bamaga et al., 2011)

\subsubsection{Hexavalent Phosphazene Salts}

Phosphazenes are hybrid organic-inorganic materials whose structure is centred on phosphorous and nitrogen and the phosphazene system has some advantages such as 
hydrolytic stability and versatile chemistry. Therefore, a novel draw solute based on the phosphazene molecule has been recently proposed and initially investigated (Stone et al. 2013b). In their study, trimeric phosphazene carboxylic acids were treated with either $\mathrm{NaOH}$ or $\mathrm{LiOH}$ to yield stable and highly water soluble sodium and lithium phasphazene salts. As a result, the Li salt showed higher initial fluxes ( $\sim \mathrm{LMH})$ as compared to the Na salt ( $\sim 6 \mathrm{LMH})$ at identical $0.067 \mathrm{M}$ DS concentrations at $30^{\circ} \mathrm{C}$. In addition, when the water flux was measured at $50^{\circ} \mathrm{C}$, the initial water flux increased ( 8.5 LMH). However, hydrolysis of the CTA membrane in some degree was observed, probably due to higher $\mathrm{pH}$ of the phosphazene DS ( $\mathrm{pH}=\sim 8)$.

\subsubsection{Polymer-Graphene Composite}

Grapene is a two-dimensional carbon material with outstanding properties thus reduced graphene oxide (rGO)-composite hydrogels were used as draw agents to enhance water flux in FO desalination (Zeng et al. 2013). The DS was prepared by combining rGO and two different hydrogels. The amounts of rGO ranged from $0.3 \mathrm{wt} \%$ to $3 \mathrm{wt} \%$, and poly (sodium acrylate) (PSA) and poly (sodium acrylate)-poly (Nisopropylacrylamide) (PSA-NIPAM) hydrogel draw agents were used in this study. The water fluxes for the pure polymer hydrogels (3.1 LMH for PSA and 1.6 LMH for PSANIPAM) were significantly smaller than a water flux of $6.8 \mathrm{LMH}(1.2 \mathrm{wt} \% \mathrm{rGO}+\mathrm{PSA}-$ NIPAM as DS) and 8.2 LMH (1.2 wt\% rGO + PSA as DS) in the FO process. In addition, although these water fluxes of the two composite hydrogels with $1.2 \mathrm{wt} \% \mathrm{rGO}$ were significantly higher than other emerging draw agents such as MNP, the results were relatively lower than more traditional draw agents such as $1.5 \mathrm{M} \mathrm{NH}_{4} \mathrm{HCO}_{3} \mathrm{DS}$ The water recovery rate in the solar dewatering process for composites with $1.2 \mathrm{wt} \%$ rGO was found to be twice as fast as that for pure hydrogels (i.e. $21 \%$ for pure PSANIPAM, 45\% for PSA-NIPAM with $1.2 \mathrm{wt} \% \mathrm{rGO}$ ).

\subsection{Concluding Remarks}

This chapter discusses an overview of the presently available DS. It also provides some general criteria for the selection of suitable DS. Based on the current literature, there is no particular DS that can be regarded as "ideal" and the selection of a suitable DS mainly depends on the targeted application. The success of most FO applications also relies on how the DS can be recovered from the produced water. Therefore, the commercialised FO processes such as FO followed by RO seawater desalination, emergency drinks and osmotic dilution are utilised without a DS recovery system, which leads to its simplicity and significant savings of energy. Research is still needed in this area and further study need to be carried out in the development of more suitable DS to achieve full-scale commercialisation of the FO process. 


\subsection{Abbreviations}

$\begin{array}{ll}\text { FO } & \text { forward osmosis } \\ \text { DS } & \text { draw solution } \\ \text { RSF } & \text { reverse salt flux } \\ \text { RO } & \text { reverse osmosis } \\ \text { MNPs } & \text { magnetic nanoparticles } \\ \text { SPS } & \text { switchable polarity solvents } \\ \text { UF } & \text { ultrafiltration } \\ \text { NF } & \text { nanofiltration }\end{array}$

\subsection{References}

Achilli, A., Cath, T. Y., and Childress, A. E. (2010). "Selection of inorganic-based draw solutions for forward osmosis applications." Journal of Membrane Science, 364(1-2): 233-241.

Achilli, A., Cath, T. Y., Marchand, E. A., and Childress, A. E. (2009). "The forward osmosis membrane bioreactor: A low fouling alternative to MBR processes." Desalination, 239(1-3): 10-21.

Adham, S., Oppenheimer, J., Liu, L., and Kumar, M. (2009). Dewatering Reverse Osmosis concentrate from water reuse applications using Forward Osmosis, WaterReuse Foundation.

Alnaizy, R., Aidan, A., and Qasim, M. (2013). "Copper sulfate as draw solute in forward osmosis desalination." Journal of Environmental Chemical Engineering, 1(3), 424-430.

Bai, H., Liu, Z., and Sun, D. D. (2011). "Highly water soluble and recovered dextran coated Fe3O4 magnetic nanoparticles for brackish water desalination." Separation and Purification Technology, 81(3), 392-399.

Bamaga, O. A., Yokochi, A., Zabara, B., and Babaqi, A. S. (2011). "Hybrid FO/RO desalination system: Preliminary assessment of osmotic energy recovery and designs of new FO membrane module configurations." Desalination, 268, 163169.

Batchelder, G. (1965). "Process for the demineralization of water." US Patent 317179903.

Beaudry, E. G., and Lampi, K. A. (1990). "Membrane technology for direct osmosis concentration of fruit juices." Food Technology, 44, 121.

Bowden, K. S., Achilli, A., and Childress, A. E. (2012). "Organic ionic salt draw solutions for osmotic membrane bioreactors." Bioresource Technology, 122, 207-216. 
Cath, T., Childress, A., and Elimelech, M. (2006). "Forward osmosis: Principles, applications, and recent developments." Journal of Membrane Science, 281(1-2), 70-87.

Chekli, L., Phuntsho, S., Shon, H. K., Vigneswaran, S., Kandasamy, J., and Chanan, A. (2012). "A review of draw solutes in forward osmosis process and their use in modern applications." Desalination and Water Treatment, 43(1-3), 167-184.

Choi, Y.-J., Choi, J.-S., Oh, H.-J., Lee, S., Yang, D. R., and Kim, J. H. (2009). "Toward a combined system of forward osmosis and reverse osmosis for seawater desalination." Desalination, 247(1-3), 239-246.

Frank, B. S. (1972). "Desalination of seawater." US Patent 367089720.

Gadelha, G. A. F., and Hankins, N. P. (2011). Assessment of draw-solution performance in combination with assymetric membranes for forward osmosis. IDAWC, Perth, Australia.

Garcia-Castello, E. M., McCutcheon, J. R., and Elimelech, M. (2009). "Performance evaluation of sucrose concentration using forward osmosis." Journal of Membrane Science, 338(1-2), 61-66.

Ge, Q., Ling, M., and Chung, T.-S. (2013). "Draw Solutions for Forward Osmosis Processes: Developments, Challenges, and Prospects for the Future." Journal of Membrane Science, 447, 1-11.

Ge, Q., Su, J., Amy, G. L., and Chung, T.-S. (2012a). "Exploration of polyelectrolytes as draw solutes in forward osmosis processes." Water research, 46(4): 1318-1326.

Ge, Q., Su, J., Chung, T.-S., and Amy, G. (2011). "Hydrophilic Superparamagnetic Nanoparticles: Synthesis, Characterization, and Performance in Forward Osmosis Processes." Industrial \& Engineering Chemistry Research, 50(1), 382388.

Ge, Q., Wang, P., Wan, C., and Chung, T.-S. (2012b). "Polyelectrolyte-Promoted Forward Osmosis-Membrane Distillation (FO-MD) Hybrid Process for Dye Wastewater Treatment." Environmental science \& technology, 46(11), 62366243.

Glew, D. N. (1965). "Process for liquid recovery and solution concentration." US Patent 9, 930.

Hancock, N. T., and Cath, T. Y. (2009). "Solute coupled diffusion in osmotically driven membrane processes." Environmental Science \& Technology, 43(17), 6769-6775.

Herron, J. R., Beaudry, E. G., Jochums, C. E., and Medina, L. E. (1994). "Osmotic concentration apparatus and method for direct osmosis concentration of fruit juices." US Patent 5, 430.

Holloway, R. W. (2006). Forward osmosis for concentration of anaerobic digester centrate. M.S., University of Nevada, Reno.

HTI (2010). "Hydration bags-technology overview." <www.hydrationtech.com.> (accessed Oct. 2013).

Jessop, P. G., Heldebrant, D. J., Li, X., Eckert, C. A., and Liotta, C. L. (2005). "Green chemistry: Reversible nonpolar-to-polar solvent." Nature, 436(7054), 1102-1102. 
Jessop, P. G., Kozycz, L., Rahami, Z. G., Schoenmakers, D., Boyd, A. R., Wechsler, D., et al. (2011). "Tertiary amine solvents having switchable hydrophilicity." Green Chemistry, 13(3), 619-623.

Jessop, P. G., Mercer, S. M., and Heldebrant, D. J. (2012). "CO2-triggered switchable solvents, surfactants, and other materials." Energy \& Environmental Science, 5(6), 7240-7253.

Jessop, P. G., Phan, L., Carrier, A., Robinson, S., Dürr, C. J., and Harjani, J. R. (2010). "A solvent having switchable hydrophilicity." Green Chemistry, 12(5), 809-814.

Kessler, J. O., and Moody, C. D. (1976). "Drinking water from sea water by forward osmosis." Desalination, 18(3), 297-306.

Kravath, R. E., and Davis, J. A. (1975). "Desalination of seawater by direct osmosis." Desalination, 16(1975), 151-155.

Lay, W. C., Zhang, J., Tang, C., Wang, R., Liu, Y., and Fane, A. G. (2012). "Factors affecting flux performance of forward osmosis systems." Journal of Membrane Science, 394, 151-168.

Lee, S., Boo, C., Elimelech, M., and Hong, S. (2010). "Comparison of fouling behavior in forward osmosis (FO) and reverse osmosis (RO)." Journal of Membrane Science, 365(1-2), 34-39.

Li, D., Zhang, X., Simon, G. P., and Wang, H. (2013). "Forward osmosis desalination using polymer hydrogels as a draw agent: Influence of draw agent, feed solution and membrane on process performance." Water Research, 47(1), 209-215.

Li, D., Zhang, X., Yao, J., Simon, G. P., and Wang, H. (2011a). "Stimuli-responsive polymer hydrogels as a new class of draw agent for forward osmosis desalination." Chemical Communications, 47, 1710-1712.

Li, D., Zhang, X., Yao, J., Simon, G. P., and Wang, H. (2011b). "Stimuli-responsive polymer hydrogels as a new class of draw agent for forward osmosis desalination." Chem. Commun., 47(6), 1710-1712.

Li, D., Zhang, X., Yao, J., Zeng, Y., Simon, G. P., and Wang, H. (2011c). "Composite polymer hydrogels as draw agents in forward osmosis and solar dewatering." Soft Matter, 7(21), 10048-10056.

Ling, M. M., and Chung, T.-S. (2011a). "Desalination process using super hydrophilic nanoparticles via forward osmosis integrated with ultrafiltration regeneration." Desalination, 278, 194-202.

Ling, M. M., and Chung, T.-S. (2011b). "Novel dual-stage FO system for sustainable protein enrichment using nanoparticles as intermediate draw solutes." Journal of Membrane Science, 372(1-2), 201-209.

Ling, M. M., Chung, T.-S., and Lu, X. (2011). "Facile synthesis of thermosensitive magnetic nanoparticles as "smart" draw solutes in forward osmosis." Chemical Communications, 47(38), 10788-10790.

Ling, M. M., and Chung, T. S. (2012). "Surface-ionized nanoparticles draw solutes and their regeneration in an integrated electric field and nanofiltration system." Industrial \& Engineering Chemistry Research, 51, 15463-15471. 
Ling, M. M., Wang, K. Y., and Chung, T.-S. (2010). "Highly Water-Soluble Magnetic Nanoparticles as Novel Draw Solutes in Forward Osmosis for Water Reuse." Industrial \& Engineering Chemistry Research, 49(12), 5869-5876.

Mamisaheby, S., Phuntsho, S., Shon, H., Lotfi, F., and Kim, J. (2012). "Factors Affecting the Performances of Forward Osmosis Desalination Process." Procedia Engineering, 44, 1449-1451.

Martinetti, C. R., Childress, A. E., and Cath, T. Y. (2009). "High recovery of concentrated RO brines using forward osmosis and membrane distillation." Journal of Membrane Science, 331, 31-39.

McCormick, P., Pellegrino, J., Mantovani, F., and Sarti, G. (2008). "Water, salt, and ethanol diffusion through membranes for water recovery by forward (direct) osmosis processes." Journal of Membrane Science, 325(1), 467-478.

McCutcheon, J. R., McGinnis, R. L., and Elimelech, M. (2005). "A novel ammonia-carbon dioxide forward (direct) osmosis desalination process." Desalination, 174, $1-11$.

McCutcheon, J. R., McGinnis, R. L., and Elimelech, M. (2006). "Desalination by ammonia-carbon dioxide forward osmosis: Influence of draw and feed solution concentrations on process performance." Journal of Membrane Science, 278, 114-123.

McGinnis, R. (2002). Osmotic desalination process. US patent 7,560,029. 1 Feb. 2002.

McGinnis, R. L., McCutcheon, J. R., and Elimelech, M. (2007). "A novel ammoniacarbon dioxide osmotic heat engine for power generation." Journal of Membrane Science, 305(1-2), 13-19.

Neff, R. A. (1964). Solvent extractor, Google Patents.

Ng, H. Y., and Tang, W. (2006). "Forward (Direct) Osmosis: A novel and prospective process for brine control." Water Environment Foundation, 4345-4352.

Ng, H. Y., Tang, W., and Wong, W. S. (2006). "Performance of Forward (Direct) Osmosis Process: Membrane Structure and Transport Phenomenon." Environ. Sci. Technol., 40(7), 2408-2413.

Noh, M., Mok, Y., Lee, S., Kim, H., Lee, S. H., Jin, G.-w., et al. (2012). "Novel lower critical solution temperature phase transition materials effectively control osmosis by mild temperature changes." Chemical Communications, 48(32), 3845-3847.

Ou, R., Wang, Y., Wang, H., and Xu, T. (2013). "Thermo-sensitive polyelectrolytes as draw solutions in forward osmosis process." Desalination, 318(0), 48-55.

Petrotos, K. B., Quantick, P., and Petropakis, H. (1998). "A study of the direct osmotic concentration of tomato juice in tubular membrane " module configuration. I. The effect of certain basic process parameters on the process performance." Journal of Membrane Science, 150(1), 99-110.

Phuntsho, S., Shon, H. K., Hong, S. K., Lee, S. Y., and Vigneswaran, S. (2011). "A novel low energy fertilizer driven forward osmosis desalination for direct fertigation: evaluating the performance of fertilizer draw solutions." J. Membr. Sci., 375(2011), 172-181. 
Phuntsho, S., Shon, H. K., Majeed, T., El Saliby, I., Vigneswaran, S., Kandasamy, J., et al. (2012). "Blended fertilizers as draw solutions for fertilizer-drawn forward osmosis desalination." Environmental Science \& Technology, 46(8), 4567-4575.

Stache, K. (1989). Apparatus for transforming sea water, brackish water, polluted water or the like into a nutritious drink by means of osmosis. US Patent 4, 030.

Stone, M. L., Rae, C., Stewart, F. F., and Wilson, A. D. (2013a). "Switchable polarity solvents as draw solutes for forward osmosis." Desalination, 312, 124-129.

Stone, M. L., Wilson, A. D., Harrup, M. K., and Stewart, F. F. (2013b). "An initial study of hexavalent phosphazene salts as draw solutes in forward osmosis." Desalination, 312, 130-136.

Su, J., Chung, T.-S., Helmer, B. J., and de Wit, J. S. (2012). "Enhanced double-skinned FO membranes with inner dense layer for wastewater treatment and macromolecule recycle using Sucrose as draw solute." Journal of Membrane Science, 396, 92-100.

Tan, C. H., and Ng, H. Y. (2010). "A novel hybrid forward osmosis - nanofiltration (FO-NF) process for seawater desalination: Draw solution selection and system configuration." Desalination and Water Treatment, 13, 356-361.

Thompson, N. A., and Nicoll, P. G. (2011). Forward osmosis desalination: A commercial reality. International Desalination Association World Congress. Perth, Australia.

Van’t Hoff, J. H. (1887). "Die Rolle der osmotischen Druckes in der Analogie zwischen Lo“sungen und Gasen." Z. Phys. Chem., 1, 481-508.

Wallace, M., Cui, Z., and Hankins, N. P. (2008). "A thermodynamic benchmark for assessing an emergency drinking water device based on forward osmosis." Desalination, 227(1-3), 34-45.

Wang, K. Y., Ong, R. C., and Chung, T.-S. (2010). "Double-Skinned Forward Osmosis Membranes for Reducing Internal Concentration Polarization within the Porous Sublayer." Industrial \& Engineering Chemistry Research, 49(10), 4824-4831.

Warne, B. B., R., Mayes, E., Oriard, T., Norris, I. (2010). Water purification method using a field separable osmotic agent. GB2464956.

WHO (2011). "Guidelines for drinking-water quality." 4th ed., <http://www.who.int/ water_sanitation_health/dwq/guidelines/en/.> (accessed Oct. 2013).

Wrolstad, R. E., McDaniel, M. R., Durst, R. W., Micheals, N., Lampi, K. A., and Beaudry, E. G. (1993). "Composition and sensory characterization of red raspberry juice concentrated by direct-osmosis or evaporation." Journal of Food Science, 58, 633-637.

Xu, Y., Peng, X., Tang, C. Y., Fu, Q. S., and Nie, S. (2010). "Effect of draw solution concentration and operating conditions on forward osmosis and pressure retarded osmosis performance in a spiral wound module." Journal of Membrane Science, 348(1-2), 298-309.

Yaeli, J. (1992). "Method and apparatus for processing liquid solutions of suspensions particularly useful in the desalination of saline water." US Patent 509857524. 
Yen, S. K., Mehnas Haja N, F., Su, M., Wang, K. Y., and Chung, T.-S. (2010). "Study of draw solutes using 2-methylimidazole-based compounds in forward osmosis." Journal of Membrane Science, 364(1-2), 242-252.

Yong, J. S., Phillip, W. A., and Elimelech, M. (2012). "Coupled reverse draw solute permeation and water flux in forward osmosis with neutral draw solutes." Journal of Membrane Science, 392, 9-17.

York, R. J., Thiel, R. S., and Beaudry, E. G. (1999). Full-scale experience of direct osmosis concentration applied to leachate management. Seventh International Waste Management and Landfill Symposium.

Zeng, Y., Qiu, L., Wang, K., Yao, J., Li, D., Simon, G. P., et al. (2013). "Significantly enhanced water flux in forward osmosis desalination with polymer-graphene composite hydrogels as a draw agent." RSC Advances, 3(3), 887-894.

Zhao, S., and Zou, L. (2011). "Effects of working temperature on separation performance, membrane scaling and cleaning in forward osmosis desalination." Desalination, 278(1-3), 157-164.

Zhao, S., Zou, L., and Mulcahy, D. (2011). "Effects of membrane orientation on process performance in forward osmosis applications." Journal of Membrane Science, 382(1), 308-315.

Zou, S., Gu, Y., Xiao, D., and Tang, C. Y. (2011). "The role of physical and chemical parameters on forward osmosis membrane fouling during algae separation." Journal of Membrane Science, 366(1-2), 356-362. 